\title{
VHDL-AMS Simulation Framework for Molecular-FET Device-to-Circuit Modeling and Design
}

\author{
Mariagrazia Graziano, ${ }^{1}$ Ali Zahir, ${ }^{2}$ Malik Ashter Mehdy ${ }^{(D},{ }^{1}$ and Gianluca Piccinini ${ }^{1}$ \\ ${ }^{1}$ Electronics and Telecommunication Department of Politecnico di Torino, Torino, Italy \\ ${ }^{2}$ Department of Electrical Engineering of the COMSATS Institute of Information Technology, Abbottabad, Pakistan \\ Correspondence should be addressed to Malik Ashter Mehdy; malik.mehdy@polito.it
}

Received 8 August 2017; Revised 31 October 2017; Accepted 7 November 2017; Published 1 January 2018

Academic Editor: Gerard Ghibaudo

Copyright (c) 2018 Mariagrazia Graziano et al. This is an open access article distributed under the Creative Commons Attribution License, which permits unrestricted use, distribution, and reproduction in any medium, provided the original work is properly cited.

\begin{abstract}
We concentrate on Molecular-FET as a device and present a new modular framework based on VHDL-AMS. We have implemented different Molecular-FET models within the framework. The framework allows comparison between the models in terms of the capability to calculate accurate $I-V$ characteristics. It also provides the option to analyze the impact of Molecular-FET and its implementation in the circuit with the extension of its use in an architecture based on the crossbar configuration. This analysis evidences the effect of choices of technological parameters, the ability of models to capture the impact of physical quantities, and the importance of considering defects at circuit fabrication level. The comparison tackles the computational efforts of different models and techniques and discusses the trade-off between accuracy and performance as a function of the circuit analysis final requirements. We prove this methodology using three different models and test them on a 16-bit tree adder included in Pentium 4 that, to the best of our knowledge, is the biggest circuits based on molecular device ever designed and analyzed.
\end{abstract}

\section{Introduction}

Emerging future nanoelectronics is being extensively explored for several applications [1-6]. More specifically molecular devices can play an important role both for sensing and for computational applications with huge advantages in terms of integration capabilities, functional density, and performance. The importance of molecular electronics has led many groups to study the transport properties of conjugated molecular structures [7-9]. Recently, molecular electronics has gained a great interest from both applied electronics and theoretical point of view $[1,2,10-16]$. Here we focus on molecular-FET (MOL-FET); however, our methods can be extended to any other molecular device. Methodologies on molecular device modeling are fragmented, it is extremely difficult to compare models, the scenario offers only a few examples on the application of MOL-FET in circuits and systems for the purpose of computation and sensing, and especially the literature does not offer a thorough analysis of impact of MOL-FET models at circuit level in terms of flexibility and computation requirements.
The novel contributions of the work to advance the state-of-the-art are the following. (1) We present a modular framework (Figures 1(a)-1(c)) based on VHDL-AMS, which is extended version of our previous work [17]. VHDL-AMS is a derivative of VHDL (VHSIC Hardware Description Language) and includes analog and mixed-signal extensions (AMS). Our modular framework enables within the same simulation engine and in the same conditions comparing different MOL-FET models at device level in terms of their capability to generate the $I-V$ characteristics comparable to those used as a reference. Here we refer to atomistic simulations in Atomistix ToolKit (ATK) $[18,19]$ as later explained (ATK Figure 4), but in general any reference data, for example, experimental values, could be included. (2) In this case, three models, developed by the authors and partially new, are being compared and discussed in the following sections: (a) a new semiempirical look-up table model (SE-LUT, Figure 5); (b) a new transmission spectrum based model (TSB Figure 7) fully described in [20] and evolved with respect to [21]; (c) FET-based equivalent model (FBE Figure 6), partially based on [22] and partially new. 


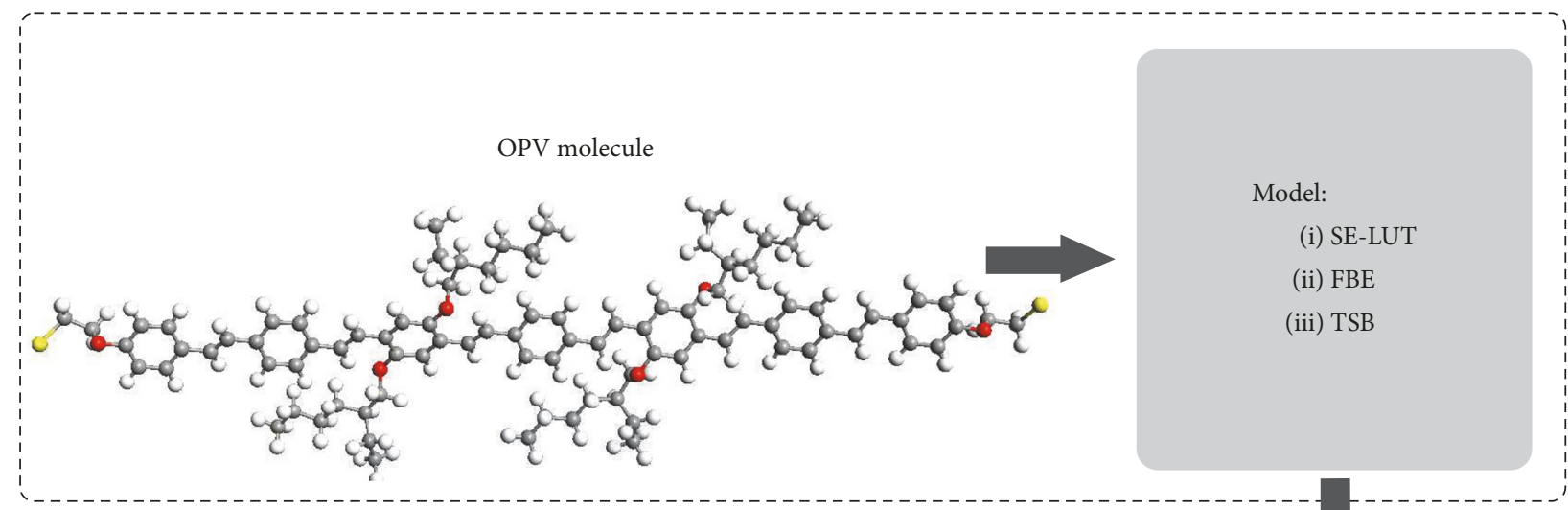

(a)

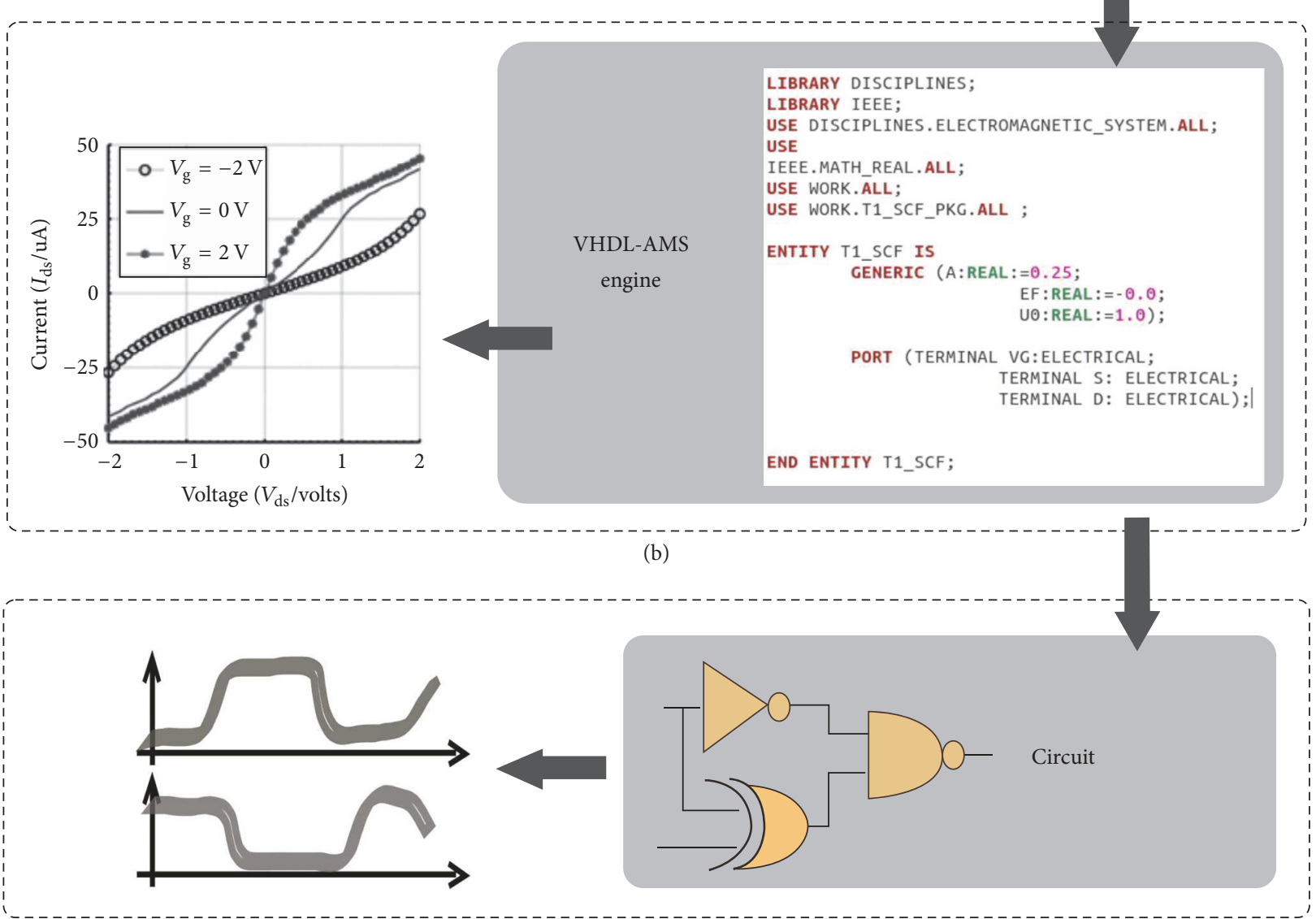

(c)

FIgURE 1: Flow of methodology. (a) The OPV molecule coupled to the electrodes through oxygen linkers is simulated in ATK for $I$-V characteristics. The conduction through the molecule is calculated at different bias conditions by the three models: semiempirical look-up table SE-LUT, transmission spectrum based (TSB) model, and FET-based equivalent model FBE. (b) VHDL-AMS based engine elaborates the results obtained by the three models, generates the corresponding $I$ - $V$ characteristics, and provides the description of MOL-FET in VHDLAMS. (c) Logic circuits based on MOL-FET can be hierarchically implemented. Correct circuit behavior and performance can be evaluated by the simulation of the circuits.

The $I-V$ characteristics from three models are compared to the reference ATK data (Figure 4) and show an excellent agreement. (3) Another fundamental novelty of this work consists in the possibility of verifying the correct MOL-FET behavior when used in a logic circuit. Several are the possible topologies; in this case we limit to a complementary P-NMOLFET crossbar that we use to design elementary logic cells. (4) The framework allows analyzing the proper circuit behavior and verifying to what extent it is possible to use each elementary logic cell in a complex architecture organized as 


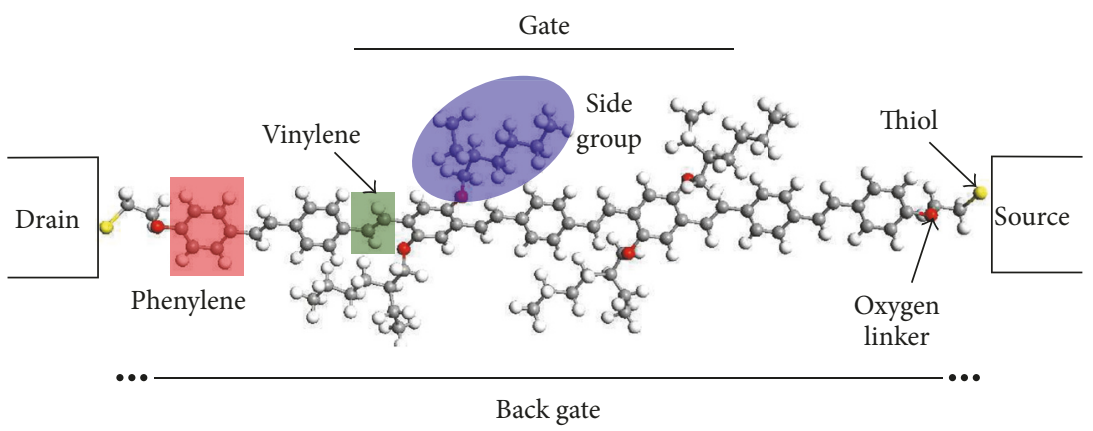

Figure 2: The structure of the device with different parts of molecule mentioned.

a cascade of basic crossbars. Here we implement as a benchmark circuit a complex up-to-date 16-bit tree adder (sparse tree used in Pentium 4 family). Steps 1 and 2 have already been presented in our previous work [17] while steps 3 and 4 are the novel contributions of this work. We also demonstrate here the capability of our methods to capture the impact of the possible variations during fabrication. Steps 3 and 4 evidence (i) whether the molecule used has the correct properties for being used in realistic circuits; (ii) the impact of the different technological choices (i.e., molecules, gate coupling) on the circuit behavior; (iii) the capabilities at circuit level offered by the different modeling features of the involved models, that is, the sensitivity to parameters and the capability to capture physical quantities, among which are the technological variations due to the immaturity and the extreme variability of molecular electronics; (iii) the models computational requirements both in terms of simulation time and in terms of setting-up of all the data and parameters necessary when a new molecule or condition is taken into consideration.

To the best of the authors' knowledge this is the first contribution allowing simulating complex circuits based on molecular devices and on a fan of possible related models whose characteristics and impact can be studied at circuit level. The rest of the paper is organized as follows: in Section 3 we present the VHDL-AMS framework while Section 4 describes the three models used. In Section 5 we use an example logic gate (EXOR) to discuss the circuit organization and its behavior and to describe the models capabilities. Section 6 finally presents the architecture of the adder and details the simulations results and discusses the performance.

\section{Device Structure and Behavior}

The molecule under study Oligo Phenylene Vinylene (OPV) is composed of repeating unit phenylene $\left(-\mathrm{C}_{6} \mathrm{H}_{4}-\right.$, the benzene ring with two hydrogen atoms removed) linked by vinylene $\left(-\mathrm{C}_{2} \mathrm{H}_{2}-\right.$, ethene with two hydrogen atoms removed) (Figure 2). The electrons are delocalized over the length of molecule because of its conjugated structure so these molecules are efficient in charge transport. The conduction depends on the length of the molecule and anchoring group between the molecule and electrodes. The side groups do not have noticeable impact on the transport properties of this device [23]. We have used thiol as anchoring group to connect with $\mathrm{Au}$ electrodes, which is common in molecular devices. The oxygen in alkyl-oxygen linker group (which links the molecule with electrode through thiol) decouples the molecule from the electrodes which results in the reduction of conductance [24]. Due to its high electronegativity, oxygen attracts the shared electrons in the polar covalent bond with its neighboring carbon atoms. So the negative charge accumulated around the oxygen leads to the decoupling between the molecule and the electrodes. We can see in Figure 4, with no gate voltage applied, a large value of drain to source bias is needed for drain current to flow (high drain to source threshold voltage). Without the linker group the conjugated molecule has good transport properties so if it was directly connected to the electrodes threshold voltage would be very low and it would behave like a molecular wire. Further information about the device chemistry can be found in $[23,24]$.

\section{Simulation Framework}

VHDL-AMS [25] is a superset of VHDL and supports the use of digital constructs together with electrical quantities, algebraic constraints, and differential equations. It allows not only the simulation of mixed-signal electronic components, but also mixed-technology systems, ranging from optics to mechanics and chemistry to thermodynamics. It is also suitable for the modeling of molecular systems.

An important property of the simulator supporting VHDL-AMS, in our case ADMS [26], is its capability of using different levels of abstraction for the description of different blocks in the system, based on the focus needed for the different blocks. This makes it possible to design using topdown methodology in which a functionality test is performed by the behavioral description of components and then, by refining the circuit, performance can be improved. ADMS [26] enhances this feature specifically as it allows the simulation of high level VHDL-AMS architectures together with the spice level netlists in the same simulation environment.

The original VHDL language allows discrete system modeling but is not capable of the description of continuous characteristics which is necessary for today's designs. The need arises in case of digital circuits when submicron effects play a role in the performance of the circuits or in case of analog and mixed-signal design. This limitation can be overcome by VHDL-AMS which in addition to the discrete features also supports the implementation of continuous 


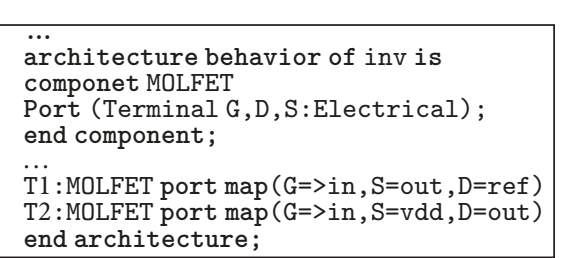

(a)

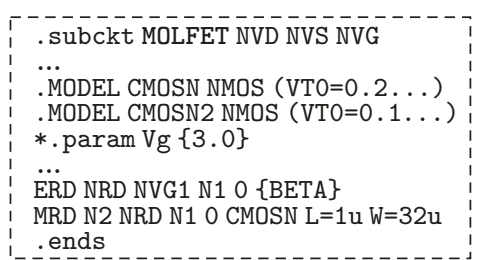

(b)

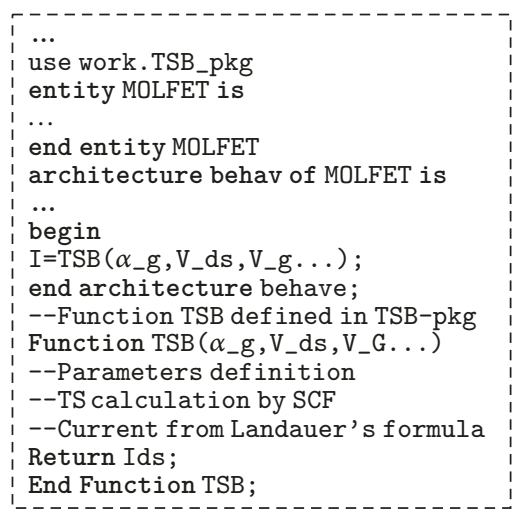

(c)

FIGURE 3: A short description of VHDL code of the framework, (a) inverter circuit implemented using the MOLFET device, (b) FBE model, and (c) TSB model description. After defining the device in models we can use it as VHDL component in any circuit ranging from inverter to $\mathrm{P} 4$ adder.

models based on mathematical equations. In [27-29] complex mixed-signal electronics and telecommunication systems have been designed using VHDL-AMS as an effective simulation language. In $[30,31]$ VHDL-AMS is suggested to model complex electronic systems with a varying hierarchical depth and interacting inside the automotive environment.

The framework based on VHDL-AMS here proposed is flexible because different MOL-FET models can be included in it as a parallel module (see the following section) and $I$ $V$ characteristics can be compared. This structure describes a circuit based on crossbar and allows the analysis of output waveforms. Depending on the type of model different features of ADMS are exploited, as the implementation of algebraic and differential equations (TSB model), the use of numerical data approximation (LUT model), or the reference to SPICE level simulation (FBE model). Once the models have been defined as the VHDL-AMS packages we can define the MOL-FET device based on these models and use it as a component in any circuit (Figure 3).

\section{MOL-FET Models}

4.1. Reference Atomistic Simulations. The MOL-FET models here adopted are referred for comparison to atomistic simulations obtained using Atomistik Toolkit [18]. The OPV molecule analyzed here has been realized and studied in [23] and it was studied in [24] to use it in an application. The molecularFET consists of dithiolated molecule with oxygen linker on both sides (Figures 1(a) and 4). This linker group decouples the molecule from electrode due to its electronegative nature which leads to narrow sharp peaks of transmission spectrum
(TS). The molecule OPV7 (OPV with seven phenylene rings) is simulated in ATK connecting the two extremes to gold electrodes as in Figure 4 named herein drain (D) and source (S) subjected to different $V$ ds bias voltages. A Gate $(V g)$ voltage is also applied, the gate being a reference substrate for the molecular system. Figure 4 on the right also shows the current-voltage characteristics of the OPV7 molecule with oxygen linkers at different gate voltages. N-type behavior in conduction can be observed as the positive gate voltage enhances the current [23]. Later the TS related to this set of simulations will also be shown and discussed in comparison with the TS model.

4.2. Semiempirical Look-Up Table (SE-LUT) Model. The method used in this case is based on a set of given $I-V$ characteristics, at different bias conditions ( $V \mathrm{~g}, V \mathrm{ds})$, obtained from atomistic simulations. The tabulated data represents the current values for different $V$ ds in rows and those for different $V \mathrm{~g}$ in columns (see Figure 5). For every gate voltage, the bias voltage is changed by a constant step of $0.04 \mathrm{~V}$. Of course the smaller the step the higher the number of simulations to be obtained but also the bigger the final resolution achieved. The set of current values for different bias points and gate voltages is provided as the input to simulation engine. The simulation engine finds the current value corresponding to the specific $V$ ds and $V$ g values in the table using a binary search algorithm. For the intermediate values of external voltages which are not present in the table, current is computed using bilinear interpolation. Due to the nature of $I-V$ function for this device, we could prove that $I-V$ characteristics can be well reproduced, using bilinear 


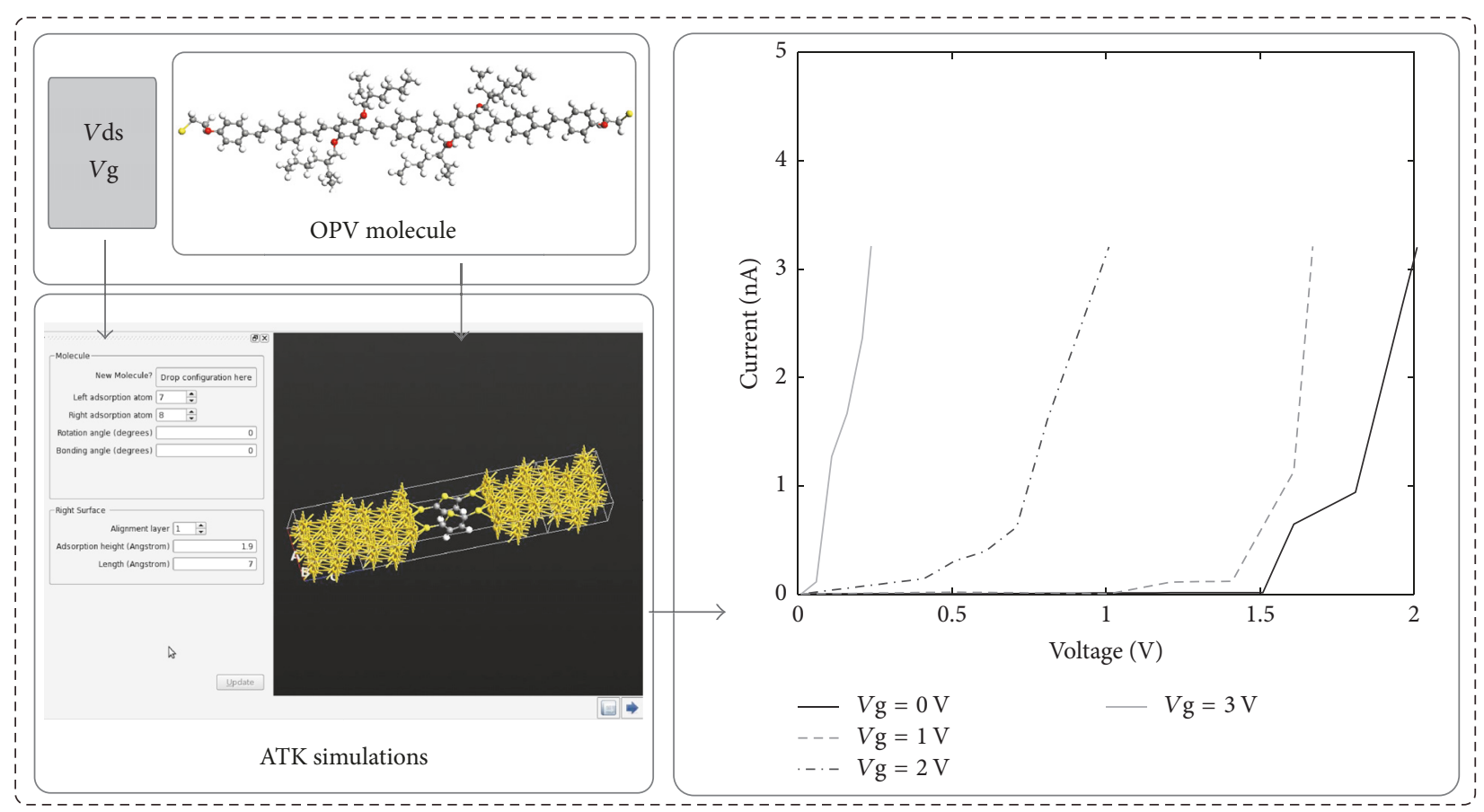

FIGURE 4: The current-voltage characteristics: given ATK simulation obtained at different bias voltages ( $V$ ds) and gate voltages ( $V \mathrm{~g})$ for OPV7 molecule.

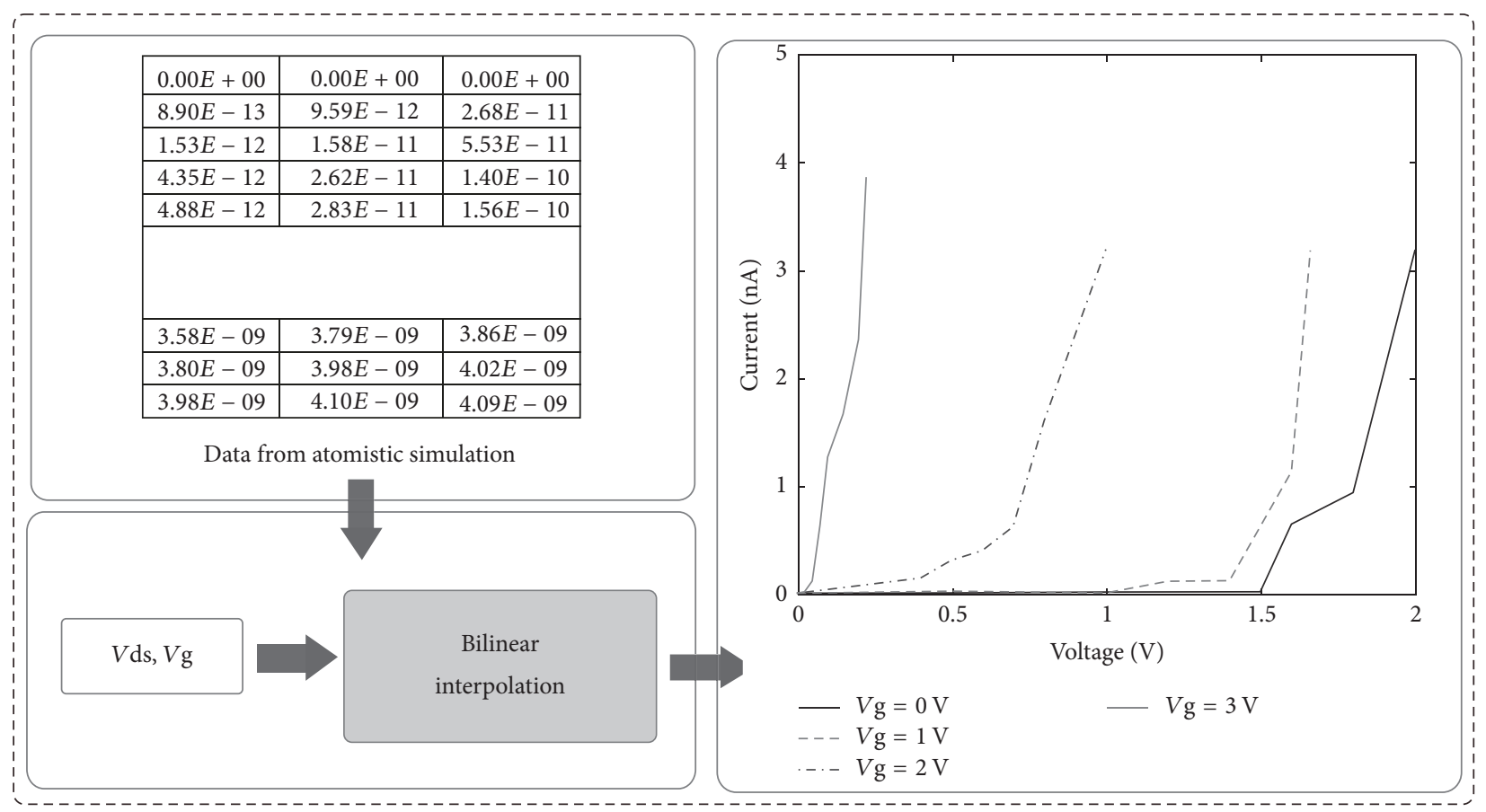

FIGURE 5: Semiempirical look-up table (SE-LUT): results obtained from ATK simulations for specific bias points $(V \mathrm{~g}, \mathrm{Vds})$ organized in tabular form; at the intermediate bias points current is obtained by bilinear interpolation.

interpolation. This model has the advantage of simplicity and can be easily implemented but it does not provide any insights on physical behavior of the device. The obtained currents are coherent with the ATK reference simulations, also in cases of $V$ ds where interpolation has to be adopted.
4.3. FET Based Electrical Equivalent (FBE) Model. In this case the model is based on [22] but reckoned in this paper for input data from different source. The method is based on an equivalent circuit model as in Figure 6. In the framework this means that ADMS has a spice-like circuit description as input 


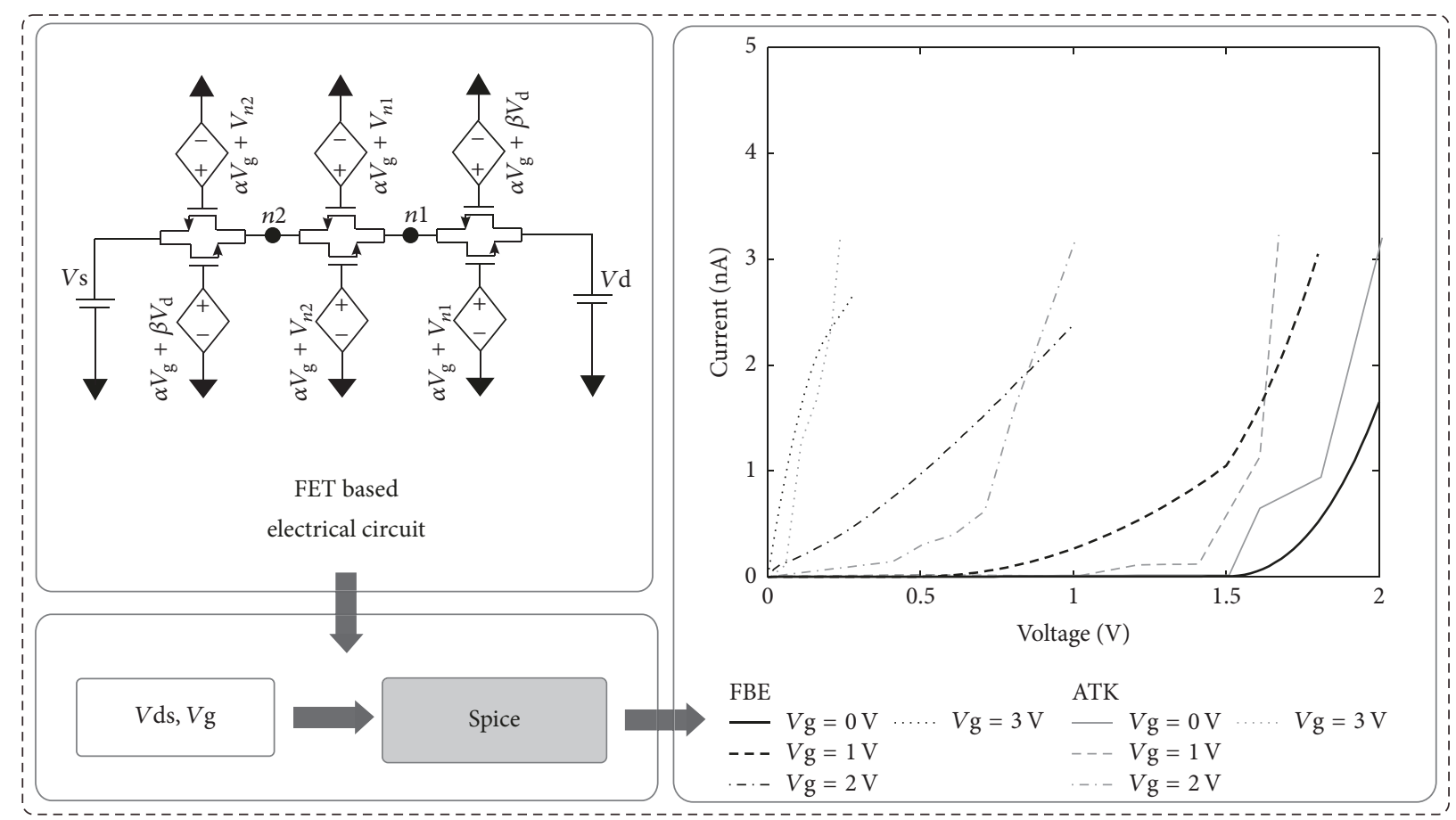

FIGURE 6: Electrical equivalent model (FBE): based on level 1 FET spice model. The phenyl ring with ethylene chain connected to it is modeled with $\mathrm{M} 1$ transistor (with $V_{\text {th }}=0.1$ ) because of delocalized pi-bonds and $\mathrm{M} 2$ transistor (with $V_{\text {th }}=0.2$ ) is used to model oxygen with only sigma bonds. Two parallel NFETs are used to model the effect of potential of neighboring atoms. Corresponding IV characteristics for different gate voltages are shown on the right (black lines: FBE; grey lines: ATK).

and calls ELDO, the spice engine included in ADMS tool, for the simulation.

The nature of the bond between atoms and external potential is two parameters which mainly influence the electron transport in the molecules. In our model these effects are represented by an equivalent circuit made of FET transistors and voltage-dependent voltage sources. The phenyl ring connected with the ethylene chain [24] is modeled with M1 transistor having $V$ th $=0.1$ because of the delocalized pibonds in Figure 6. And a high electronegative oxygen atom with only sigma bonds is modelled with $\mathrm{M} 2$ transistor $(V$ th $=$ 0.2 ), values adopted from [22]. In both cases, in order to take into account the effect of potential of neighboring atoms in a chain, two identical parallel NFETs are adopted. A voltage divider models the insulating behavior of alkane chains. The gate to molecule electrostatic coupling is introduced into the model by a coupling coefficient herein referred to as $\alpha$ (see [22] for further details on the different contributions). The $I-V$ curves are shown in Figure 6 on the right, although the shape of the curves are not very similar to ATK results but it gives acceptable results in terms of order of magnitude of the currents. As the simulation engine uses lumped circuit elements to model the current so some differences with respect to ATK can be observed in the $I-V$ characteristics. In the results sections further discussion on the effectiveness of this method will be given.

With respect to the LUT model, this equivalent model provides us with better insights on the physical behavior of the MOL-FET. For example, the gate coupling parameter $\alpha$ is not present in the SE-LUT model, where, if a different coupling has to be considered, a new set of simulations or of measurements should be obtained and loaded in the system. This partial increment of flexibility is obtained at the price of a more complex description. Moreover, an initial fitting process is required to detect the electrical equivalence. If, then, a different molecule or different linkers or the presence of variations due to technology should be taken into account, then a new fitting procedure should be done. Furthermore, this modeling technique requires using a spice-level engine during the simulation that could increase the total simulation time with respect to the pure LUT method. The model can be improved by including more circuit elements to take into account more complex chemical and physical effects.

4.4. Transmission Spectrum Based (TSB) Model. The model in this case involves an analytical approach; that is, it requires the implementation of a set of functions in the VHDL-AMS framework. The idea is sketched in Figure 7. First of all, the transmission spectrum (TS) at equilibrium is calculated. Semiempirical Extended Huckel Theory (SE-EHT) is used to find the TS and other transport properties. Atomistic simulations (in this case using ATK) at equilibrium are carried out once for all. The TS are numerical inputs for the VHDLAMS framework. These parameters are then used during the simulation execution to estimate voltage-dependent TS at nonequilibrium conditions. The model functions are called during the simulation and on the basis of the bias conditions will output the expected currents. The model is an extended 


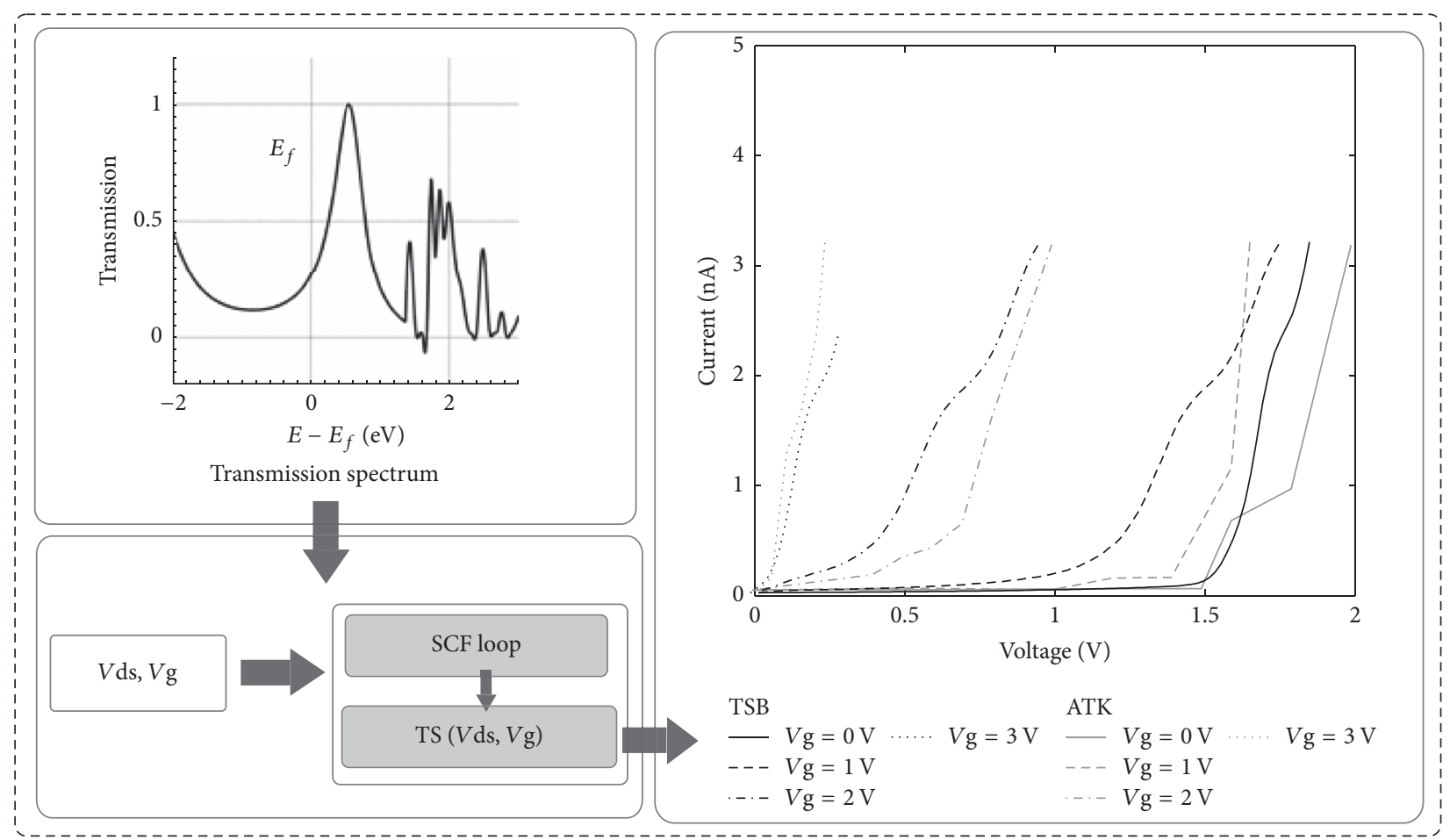

FIGURE 7: Transmission spectrum based (TSB) model: based on a set of equations to compute the quantum transport inside the molecule. The transmission spectrum (TS) at equilibrium is obtained from ATK simulations and given as input to the model. For nonequilibrium conditions, that is, external voltages applied $(V \mathrm{~g}, V \mathrm{ds})$, self-consistent field calculations are performed to find the TS and then current is calculated using TS.

version with respect to the one presented in [21]. The charge distribution in the molecule is dependent on the external voltage and changes when voltage is applied. When an external voltage is applied, the charge distribution in the molecular system changes. This causes the modification in terms of shift in the molecular energy levels and thus the TS as it is clear from Figures 8 and 9 where a comparison between this model and atomistic simulations is given in terms of TS. Thus charging effect should be taken into consideration to compute the shift in the TS. In TSB model, self-consistent field method (SCF) is used to estimate the shift in energy levels [1]. After calculating the new TS based on the shifted energy levels, Landauer's formula is used to calculate current from TS. A few assumptions are made: we assume (i) a linear shift in the energy levels at low applied voltages and (ii) that the charging effect modifies all the energy levels uniformly and TS is shifted rigidly on the energy axis. We tested the system in several conditions and verified that these assumptions can efficiently reproduce the transport behavior of the molecular system without losing the accuracy of the system (a detailed discussion on the theory and demonstration of this model is out of the scope of this paper and can be found in our previous paper [20]). The resulting $I-V$ characteristics are shown in Figure 7 on the right. The shape of the IV curves depends strongly on the position of transmission peaks and the area under the peaks. So it is a little different from ATK as the small error in the peaks position and shape can change the magnitude of the current. The shape of the curves from this model is better than the FBE model and the magnitude of current is also very close to ATK results.

If we compare this model to the previous ones it is easy to understand that this model captures with more details the physical characteristics (see the results section) and provides the option to explore the MOL-FET behavior in different physical conditions. The cost for this model is an initial extraction and analysis of TS and a more complex description, which implies more CPU intensive calculations during circuit simulation. In future the model can be improved by implementing the subtle effects like the evolution in the width of the transmission peaks and the separation between different peaks. This will result in more accurate IV characteristics.

\section{From Transistor to Circuit}

We designed a small library of logic gates. The circuit topology here chosen is implemented as a crossbar; Figure 10 shows the example of a half adder and a full adder. We used a complementary logic based on both N-type and P-type MTs. Alternatively an N-FET plus resistors could be used but the complementary structure is preferable because of low power consumption. The P-type transistor can be realized using a slightly different procedure. The difference between P-type and $\mathrm{N}$-type is based on the molecular energy levels contributing to the conduction: in the N-type transistor the LUMO levels are involved in conduction, while for the P-type 

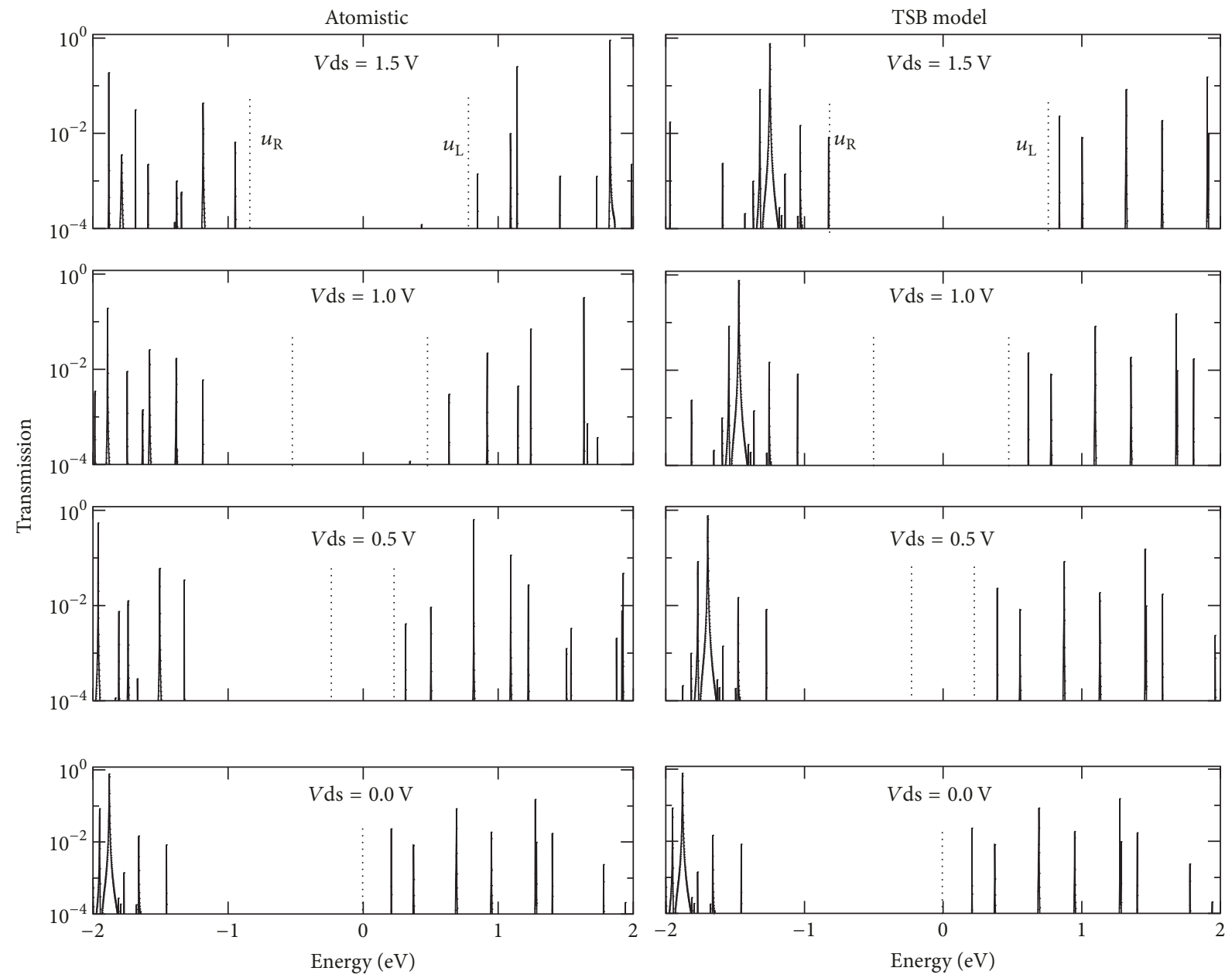

Figure 8: Transmission spectrum for different values of $V \mathrm{ds}(V g=0)$. The dotted lines represent the bias window. Transmission spectrum shifts to new energies when $V$ ds is changed. TSB model shows the same effect using self-consistent field loop.

transistor the HOMO levels contribute to conduction. For the P-type device HOMO level should be near the Fermi level. In this way, when a negative bias is applied on the gate, it shifts the energy levels towards high values and HOMO level contributes to the conduction. This issue can be addressed in two possible ways: selecting the molecule which has HOMO level near Fermi level or using a back-gate to shift the energy levels with respect to the electrodes chemical potential. We used the back gate technique with the same molecule (OPV) as used for the $\mathrm{N}$-type. When we apply an appropriate amount of back gate voltage $\left(V_{\mathrm{BG}}=-3.6 \mathrm{~V}\right)$, the energy levels shift with respect to Fermi level. The HOMO level becomes closer to Fermi level and we can use the device as P-type. The back gate is also used for $\mathrm{N}$-type device in order to tune the threshold voltage of the device. In case of N-type device we apply a back gate voltage $\left(V_{\mathrm{BG}}=1 \mathrm{~V}\right)$ to make the LUMO level so close to Fermi level that the conduction becomes possible at low bias conditions (which are stated below).

The different regions of the architectures are highlighted and mentioned in Figure 10(a). Molecular transistors and nanowires are used to construct the P-type and $\mathrm{N}$ type regions. Interconnections enable the communication between the transistors as well as between devices and inputs/outputs. Back gate electrodes for the N-type and Ptype also need interconnection space. A common back gate was used for the whole N-type region and similarly another one for P-type region Figure 10(a). Vertical and horizontal nanowires are connected using resistors at the junctions. A more complex structure like full adder Figure 10(b) is designed and simulated.

Figure 11 shows representative input/output for the input combinations of $A, B$ and $C$ in. The input signal levels are swept from logic $0(-0.3 \mathrm{~V})$ to $\operatorname{logic} 1(+0.3 \mathrm{~V})$. The value of power supply voltage is $0.3 \mathrm{~V}$ and the reference voltage is $-0.3 \mathrm{~V}$. The outputs $S$ and $C$ for all three models show that these values have very close high and low values and, moreover, these values are stable after each input changes. The corresponding truth table in Figure 11(b) demonstrates the narrow distribution of the output voltage for both low and high states. This shows the potential to further integrate the device into a larger-scale integrated circuit such as 16-bit full adder in a cascaded configuration (see the next section).

5.1. Impact of Gate Coupling. Transport properties of molecular devices can be significantly effected by the factors like molecular length, gate coupling, and coupling between metal 

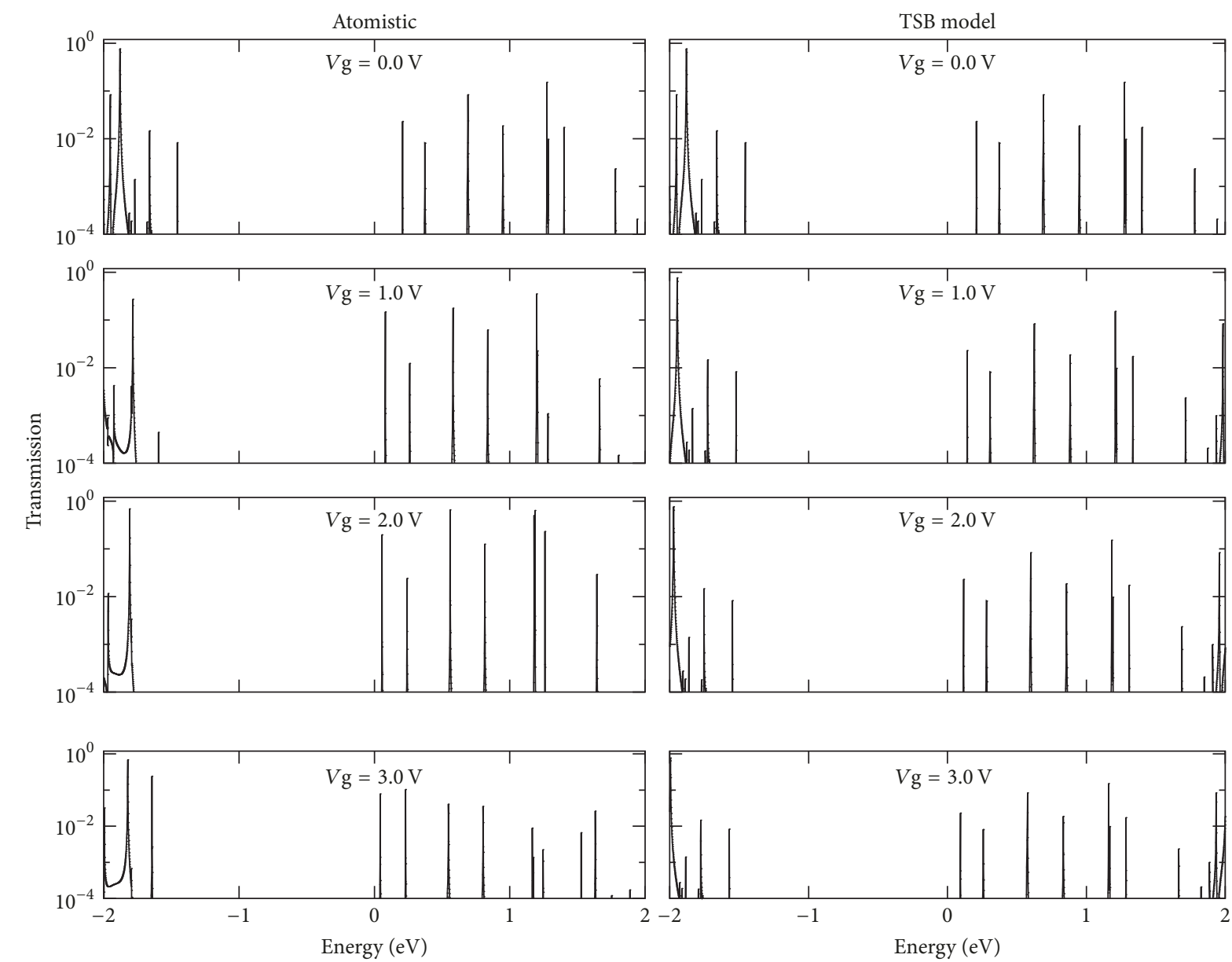

voltage.

and molecule. For the case of gate coupling we show in Figure 12 as an example the result for a simple inverter based on a structure not complementary but based on $\mathrm{N}$ FET and resistor as presented in [17] (very similar to the complementary structure, just we do not show the circuit for space reason; please refer to the cited paper for details). We show it in this case because the point is more clear than in the complementary case. The inverter shows a correct logic value at the output evaluation. But the difference can be seen in the form of high voltage level for logic 0 so cascaded gate can not be derived. A different gate coupling can be used to solve this problem. Only FBE and TSB models allow the change in gate coupling. Other physical values like slight changes in energy levels (for example due to change in environment conditions) can only be captured by TSB model by changing the TS according to the change in energy levels. But in case of FBE model the change in energy levels can not be taken into account and it would need new fitting procedure. Our methods can be used to find trade-off between the models according to the simulation requirements and conditions.

5.2. Impact of Fabrication Induced Variations. Increasing length of molecule changes the transmission spectrum (TS) in three ways: (1) the number of transmission peaks in a given energy range is increased; (2) the HOMO-LUMO gap is reduced; (3) width of transmission peaks decreases. The current through device changes as a result of these changes in TS, and thus the circuit behavior also changes. In order to include these variations in the SE-LUT model atomistic simulations for all the values of external voltages are necessary. In case of FBE model initial fitting procedure would be required. The TSB model allows including these variations easily, by analyzing TS and changing metal-molecule coupling. In the following we show some results on the impact of this type of variation.

Figure 13 shows the transmission spectrum for different length of molecule, that is, OPV3 having 3 benzene rings, OPV5 having 5 benzene rings, and OPV7 having 7 benzene rings. As expected, increasing the molecular length changes the TS in two ways. First, it increases the number of transmission peaks. This can be observed if we compare the transmission spectrum of these three molecules. The OPV7 has maximum number of peaks while OPV3 has minimum number of peaks. Second, it reduces the width of the transmission spectrum. It is shown in the encircled area (red) in the figure. The width of the transmission spectrum decreases from OPV3 to OPV7 molecule. 


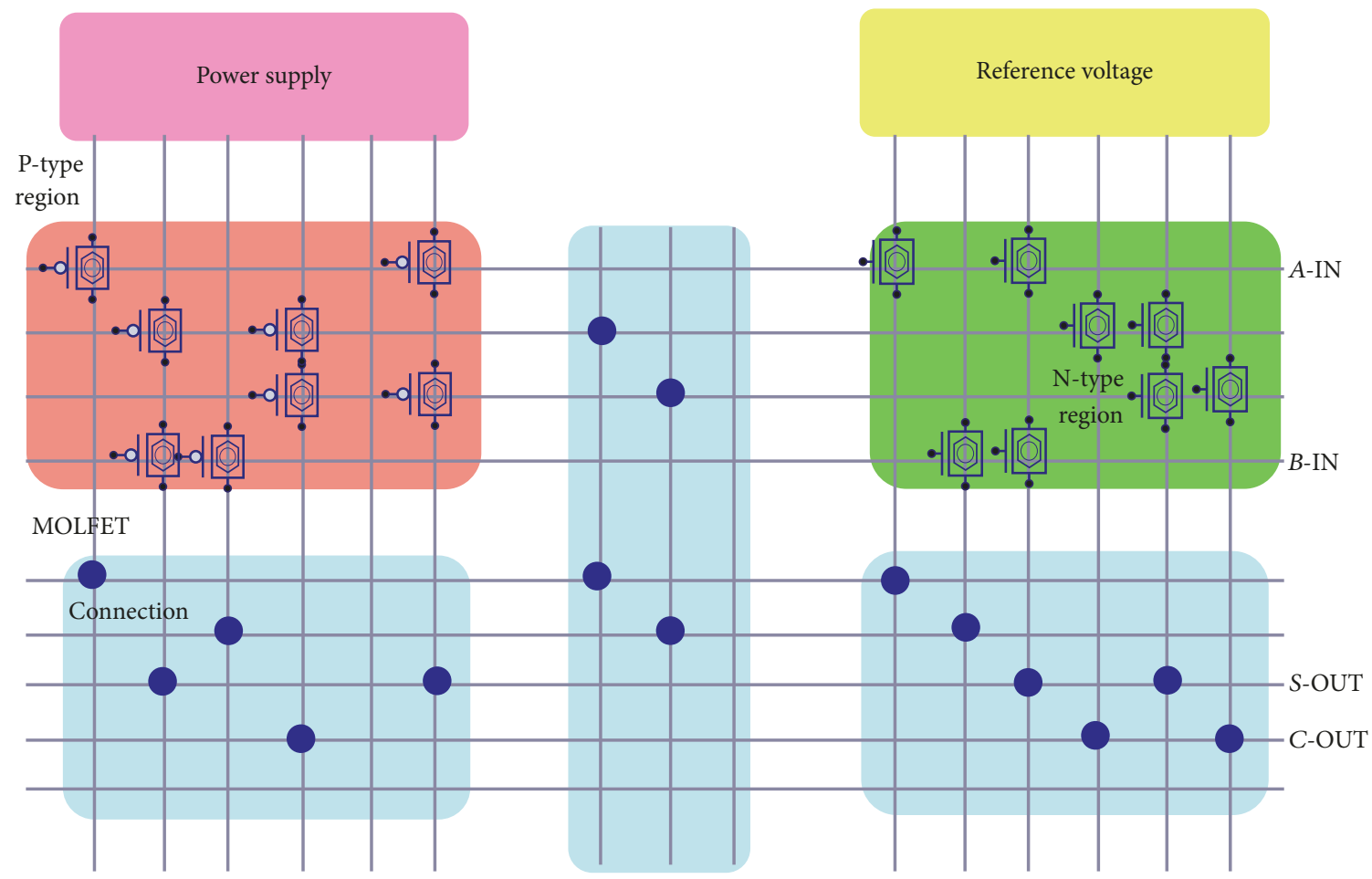

Nanowire

Interconnect and connection region

- 0

(a)

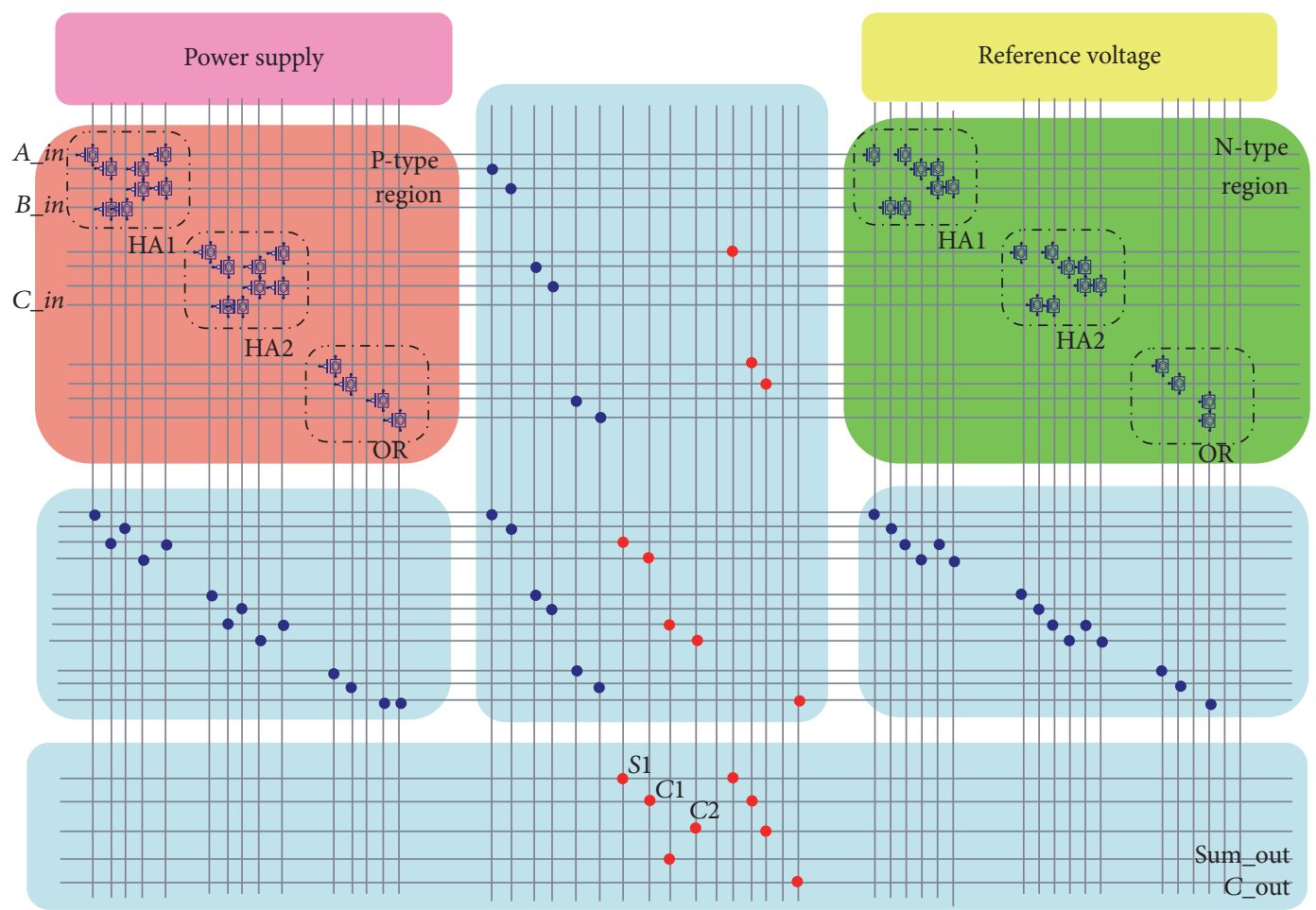

(b)

FIgURE 10: Continued. 

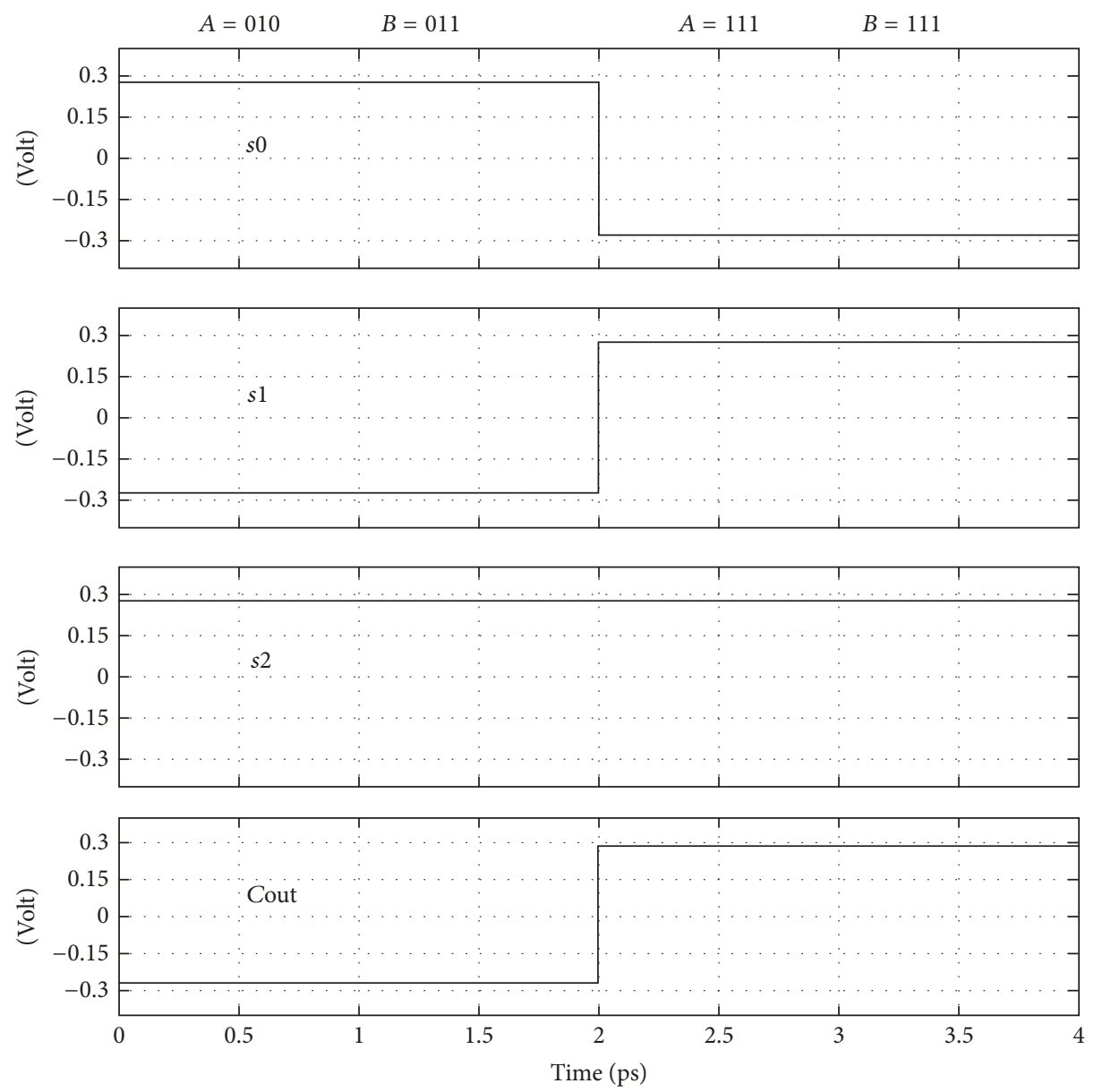

(c)

FIGURE 10: Crossbar architecture of a: (a) half adder implemented using complementary logic. The orange color shows a common back gate for P-type region and the green shows that for N-type region. (b) Single-bit full adder, implemented by the connection of two HAs and an OR gate. (c) Output waveforms of a 3-bit ripple carry adder implemented using complementary logic. In this case, the low and high input values of $-0.3 \mathrm{~V}$ and $+0.3 \mathrm{~V}$ are used to encode logic " 0 " and "1," respectively.

Figure 14 shows how the effect of changes in TS due to molecular length impacting the $I-V$ characteristics of the molecular system. The highest current is observed in OPV3 molecule and it decreases with increase in length: OPV7 has the smallest current. These simulations were obtained using ATK and the data observed from TS variations were included in the TS model. We were then able to analyze the impact of variations at the circuit level.

Figure 15 shows the implementation of the HA with data found for three different molecules: OPV3, OPV5, and OPV7. Table 1 shows the output voltages for both high and low states. In table input 0 logic represents $-0.3 \mathrm{~V}$ and input logic 1 represents $0.3 \mathrm{~V}$. As described earlier, the number of transmission peaks in a specific range increases with the increase in molecular length. So when the device is on there are more numbers of peaks within the bias window and the current increases. When device is off there are no peaks within the bias window so increased number of peaks in case of larger molecule does not increase off current. On the other hand increasing the molecular length decreases the
TABLE 1: Details on voltage levels for the HA in presence of different molecule length.

\begin{tabular}{cccccccc}
\hline Input & \multicolumn{3}{c}{ Sum } & & \multicolumn{3}{c}{ Carry } \\
B & A & OPV3 & OPV5 & OPV7 & OPV3 & OPV5 & OPV7 \\
\hline 0 & 0 & $-169 \mathrm{mV}$ & $-183 \mathrm{mV}$ & $-295 \mathrm{mV}$ & $-293 \mathrm{mV}$ & $-295 \mathrm{mV}$ & $-299 \mathrm{mV}$ \\
0 & 1 & $179 \mathrm{mV}$ & $190 \mathrm{mV}$ & $295 \mathrm{mV}$ & $-264 \mathrm{mV}$ & $-267 \mathrm{mV}$ & $-298 \mathrm{mV}$ \\
1 & 0 & $183 \mathrm{mV}$ & $193 \mathrm{mV}$ & $295 \mathrm{mV}$ & $-264 \mathrm{mV}$ & $-267 \mathrm{mV}$ & $-298 \mathrm{mV}$ \\
1 & 1 & $-195 \mathrm{mV}$ & $-206 \mathrm{mV}$ & $-195 \mathrm{mV}$ & $157 \mathrm{mV}$ & $170 \mathrm{mV}$ & $295 \mathrm{mV}$ \\
\hline
\end{tabular}

width of peaks. This leads to decrease in the current of the device with larger molecular length. This phenomenon affects both the on current and off current of the device. Overall effect of increasing the molecular length is to decrease the ofF current and the on current. But the reduction in ofF current is more than the reduction in oN. So the ratio between oN current and off current increases which improves the device performance with increase in molecular length. 

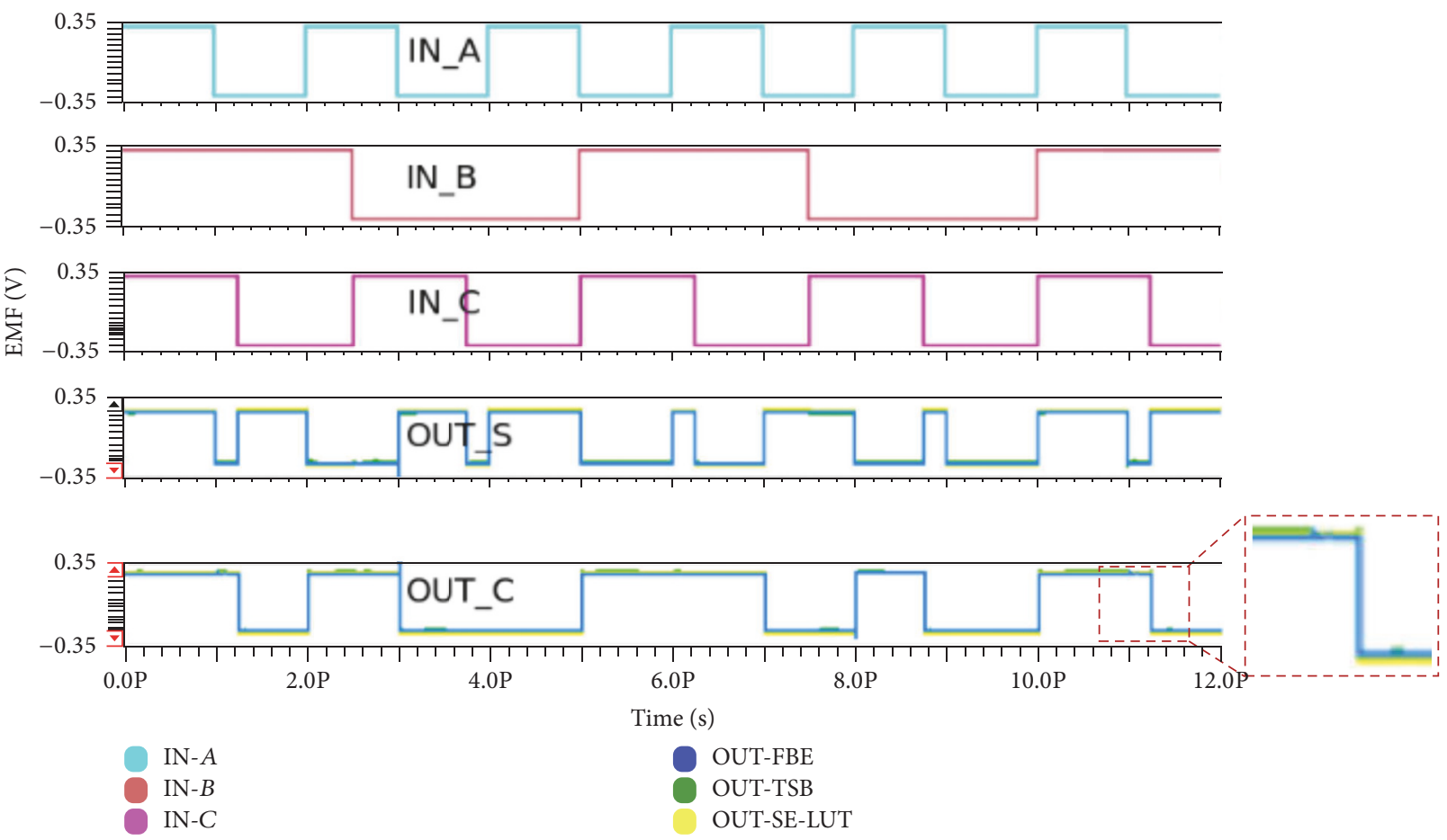

\begin{tabular}{cccccc}
\hline$A$ & $B$ & C_in & $\begin{array}{c}\text { Sum } \\
\text { TSB_Model }\end{array}$ & $\begin{array}{c}\text { Sum } \\
\text { FBE_Model }\end{array}$ & $\begin{array}{c}\text { Sum } \\
\text { FBE_Model }\end{array}$ \\
\hline 0 & 0 & 0 & 0 & 0 & 0 \\
& & & $(V:-0.222)$ & $(V:-0.240)$ & $(V:-0.235)$ \\
0 & 0 & 1 & 1 & 1 & 1 \\
& & & $(V:-0.221)$ & $(V: 0.238)$ & $(V: 0.222)$ \\
0 & 1 & 0 & $(V: 0.223)$ & $(V: 0.239)$ & $(V: 0.236)$ \\
& & & 0 & 0 & 0 \\
0 & 1 & 1 & $(V:-0.225)$ & $(V:-0.240)$ & $(V:-0.236)$ \\
& & & 1 & 1 & 1 \\
1 & 0 & 0 & $(V: 0.23)$ & $(V:-0.240)$ & $(V:-0.224)$ \\
& & & 0 & 0 & 0 \\
1 & 0 & 1 & $(V:-0.221)$ & $(V:-0.240)$ & $(V:-0.236)$ \\
& & & 0 & 0 & 0 \\
1 & 1 & 0 & $(V:-0.236)$ & $(V:-0.240)$ & $(V:-0.234)$ \\
& & & 1 & 1 & 1 \\
1 & 1 & 1 & $(V: 0.220)$ & $(V: 0.238)$ & $(V: 0.220)$ \\
\hline
\end{tabular}

FIGURE 11: Logic behavior of the full adder outputs (Sum and Carry) with respect to input $A, B$ and Carry-in. The output results of all three models are comparable: green line for TSB, blue line for SE-LUT, and yellow line for FBE.

Clearly the molecular length also affects the behavior of the circuit as the voltage levels of the circuits change with molecular length. These kinds of variations are highly important considering the infancy of this technology. Being able to easily include them in the simulation framework is fundamental to execute realistic simulations. These examples were included here just to show the importance of the point and the following section follows the mainstream of demonstrating the framework capabilities in terms of complexity of the description and of the performance evaluation. We are currently working on the extension of the framework for including process variations in a Monte-Carlo-like fashion exploiting the potentials of the TS model. Results will be presented in a future paper.

\section{Architecture}

The analysis of a complex architecture based on a cascade of crossbars can evidence (i) the effect of the different technological choices (e.g., gate coupling, molecules) on 


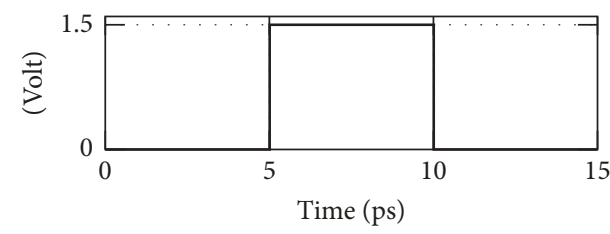

(a)

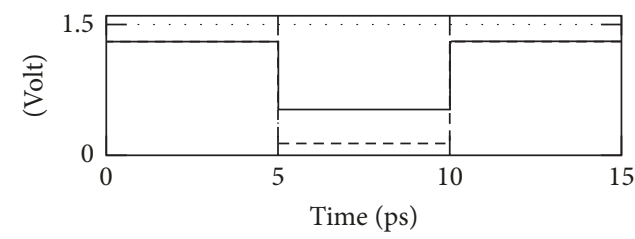

(c)

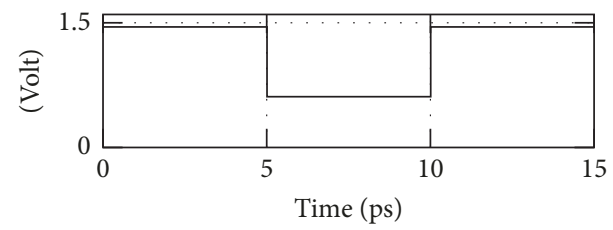

(b)

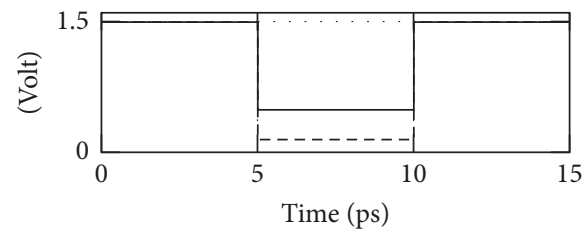

(d)

FIGURE 12: Input and output waveforms of inverter: (a) input data. Output data obtained by (b) SE-LUT models, (c) FBE model, and (d) TSB model.

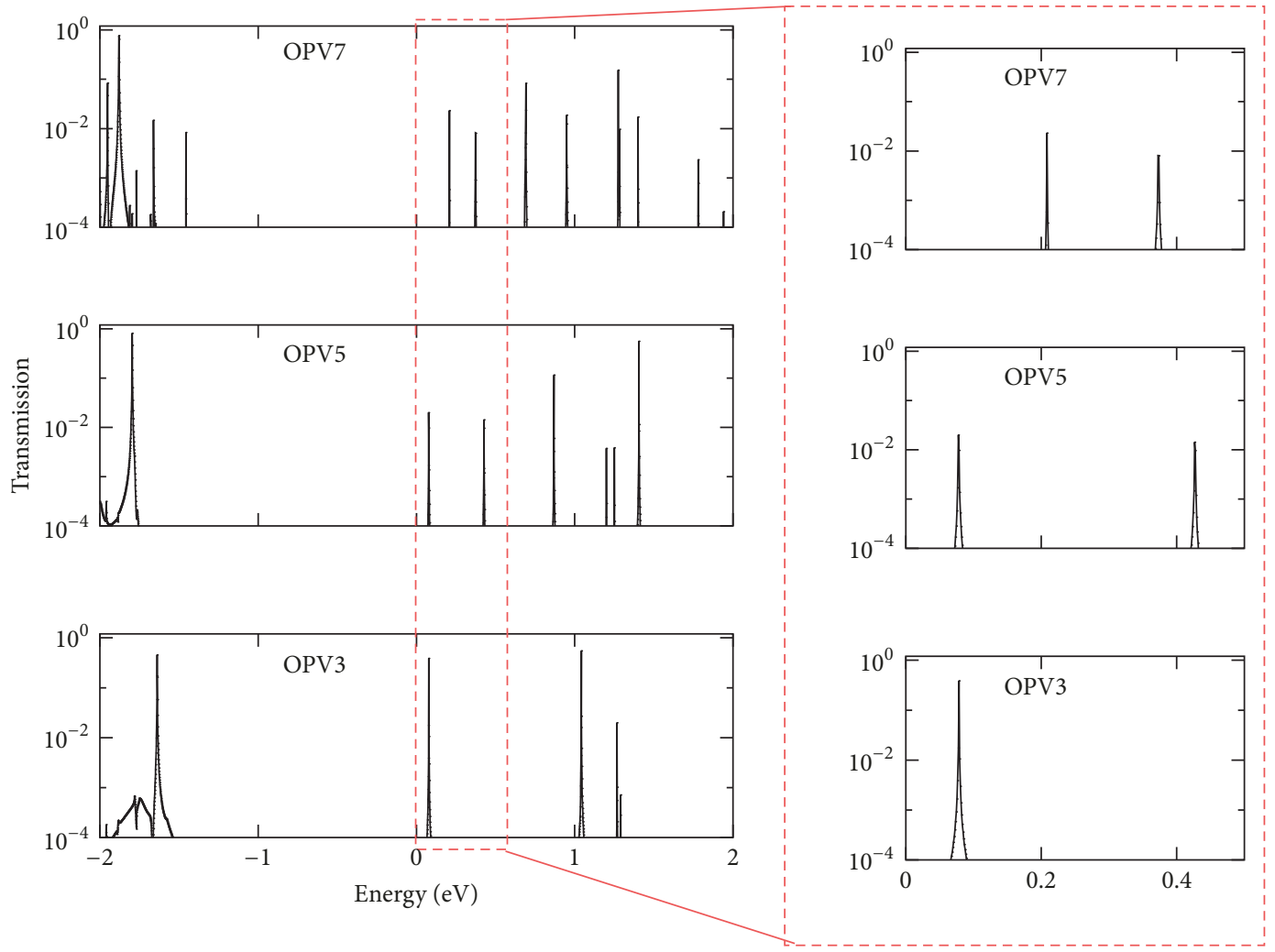

FIGURE 13: TS for molecules of different length OPV3, OP5, and OPV7.

the behavior of circuit, (ii) the capabilities provided by the different models at circuit level, and (iii) the models computational requirements.

The Pentium 4 adder is used here as reference architecture.

6.1. Pentium 4 Adder Implementation. Figure 16 shows the general block diagram of the P4 adder. It is based on two substructures: a carry select adder for sum generator and a sparse tree for carry generator. The Sum generator of 16-bit P4 adder is divided into 4 groups of 4-bit carry select adder. Each group is organized in two stacked identical 4-bit ripple carry adders (RCA), each generating 4-bit sum and an outgoing carry. One RCA assumes that the incoming carry into the group is 0 , while other assumes that it is 1 . These two RCAs generate the output in parallel. The final output of the group is selected from the value assigned by sparse tree of the carry generator by means of 4-bit multiplexers.

The carry generator block of P4 adder consists of a sparse tree as shown in Figure 16. This architecture computes every fourth carry in the adder and provides the carryin signal for individual carry select adder block, effectively 


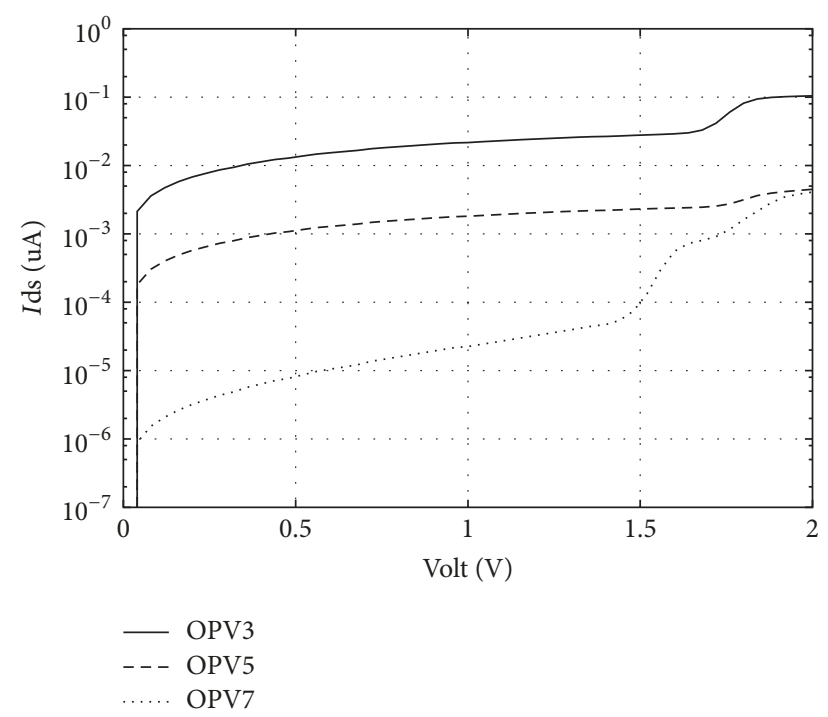

FIGURE 14: Impact of molecule length on $I-V$ curves.
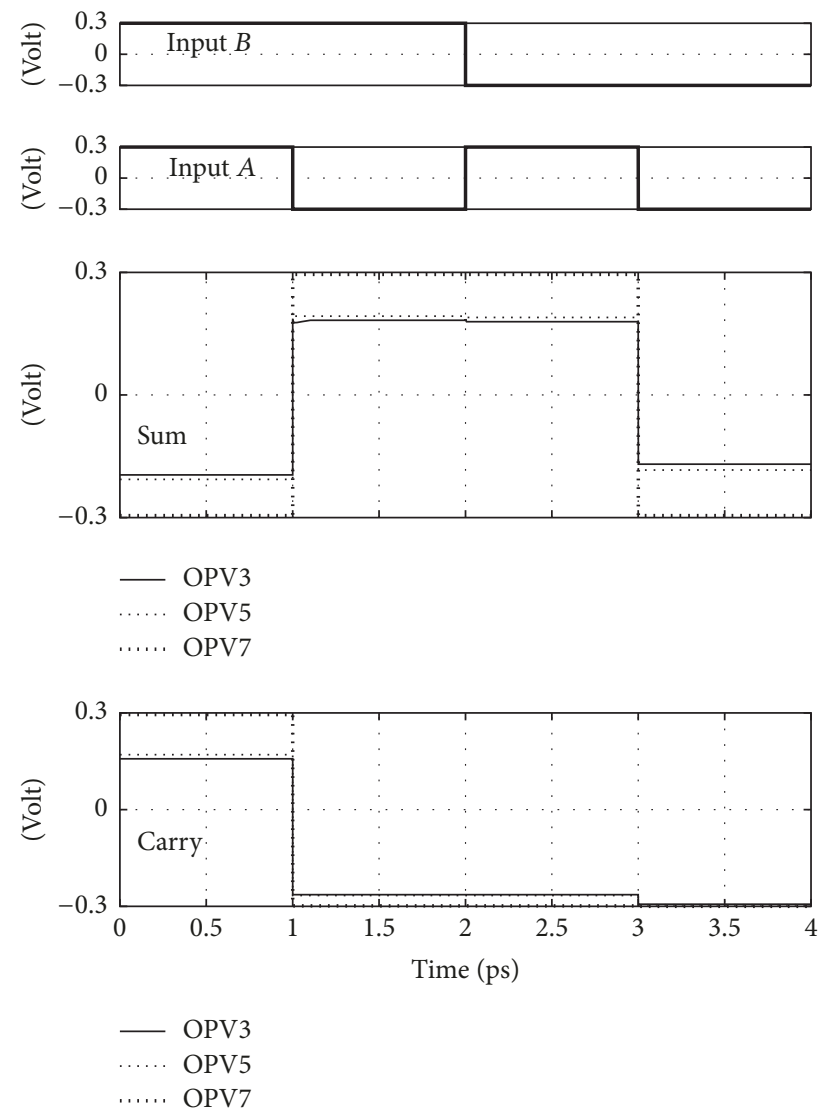

FIGURE 15: HA waveforms in presence of different molecule length.

reducing the critical path of the carry signal. In this way it is possible to work on all the carry generator groups of 4-bits without the need to wait for the carry resulting from previous groups. The tree is made up of a pseudo-regular structure of building blocks. The blocks used to implement the tree are as follows: (1) PG network: it computes propagation (Pin) and generation (Gin) signals using input signals; (2) PG block: it computes propagation $(\mathrm{Pi})$ and generation $(\mathrm{Gi})$ signals using input signals; (3) G block: it computes only generation (Gi) signals.

Figure 17 shows the output of the 4-bit carry select adder. The P4 adder is based on two substructures, carry generator and sum generator. Sum generator of 16 -bit adder is divided into 4 groups of 4 -bit carry select adder CSA. Figure 17 is used to show the proper functionality of the CSA block. The logic level of Sum [2] and sum [3] (3rd and 4th bit of sum) of three models are also shown in the figure. Good voltage levels are maintained which shows the potential to integrate the device into large scale.

For performance analysis, the result of 16-bit sparse tree and the full adder is shown in Figure 18. As an example, input values and their corresponding sum outputs are shown by red lines. Simulation results show the correct operation of the adder. The output voltage levels are also maintained. For a comparison, the output waveforms obtained by all three models are placed together which shows very small difference with respect to each other.

To analyze the voltage levels at different nodes, we select nodes N1 to N9 in the longest chain of the sparse tree as shown in Figure 19(a). Voltage level of each selected node (N1 to N9) obtained by three models is compared: TSB, SE-LUT, and FBE (Figure 19(b)). The input values are set such that on each node we get logic " 0 " output $(-300 \mathrm{mV})$. For FBE model voltage varies from $-245 \mathrm{mV}$ to $-265 \mathrm{mV}$, for TBS the variation is between $-225 \mathrm{mV}$ and $-300 \mathrm{mV}$, and for SE-LUT it is between $-215 \mathrm{mV}$ and $-295 \mathrm{mV}$. Initially, the swing of voltage level increases from moving to higher nodes. However, after node 5 the swing of voltage level becomes stable. This trend can be observed by all three models.

6.2. Timing Comparison. We here want to give a very simple identification of the difference in timing performance for the three models. Timing does not involve only the final circuit simulation time, but also the effort required to setup the model data in order for them to be included in the simulation framework. This analysis gives an idea of which model could be useful depending on the type of data available and the type of analyses requested. We compare the SE-LUT and the TSB models as they are highly different in terms of characteristics on timing and on flexibility in the final results achievable.

Time required to implement $\mathrm{P} 4$ adder is evaluated as a function of the number of bias points $(N V \mathrm{ds})$ and number of gate voltages $(\mathrm{NVg})$ points of the MOL-TRAN characteristics. For the sake of simplicity hereinafter we consider $N V \mathrm{~g}=N V \mathrm{~d} s=N$. The timing analysis of both systems is estimated as follows:

$$
\begin{aligned}
T_{\mathrm{SE}-\mathrm{LUT}} & =T_{\mathrm{Atm}} * N^{2}+T_{\mathrm{Arch}}+T_{\mathrm{Intr}}, \\
T_{\mathrm{TSB}} & =\left(2 * N * T_{\mathrm{Atm}}\right)+\left(10 * T_{\mathrm{Arch}}\right)+T_{\mathrm{Algo}} .
\end{aligned}
$$

$T_{\text {Atm }}$ is the time required by atomistic simulations. The time required to compute current at given $V$ ds and $V g$ depends on the convergence time of the self-consistence field loop. If the convergence occurs early (e.g., within 10 iterations), then the computation time would be less. And if 

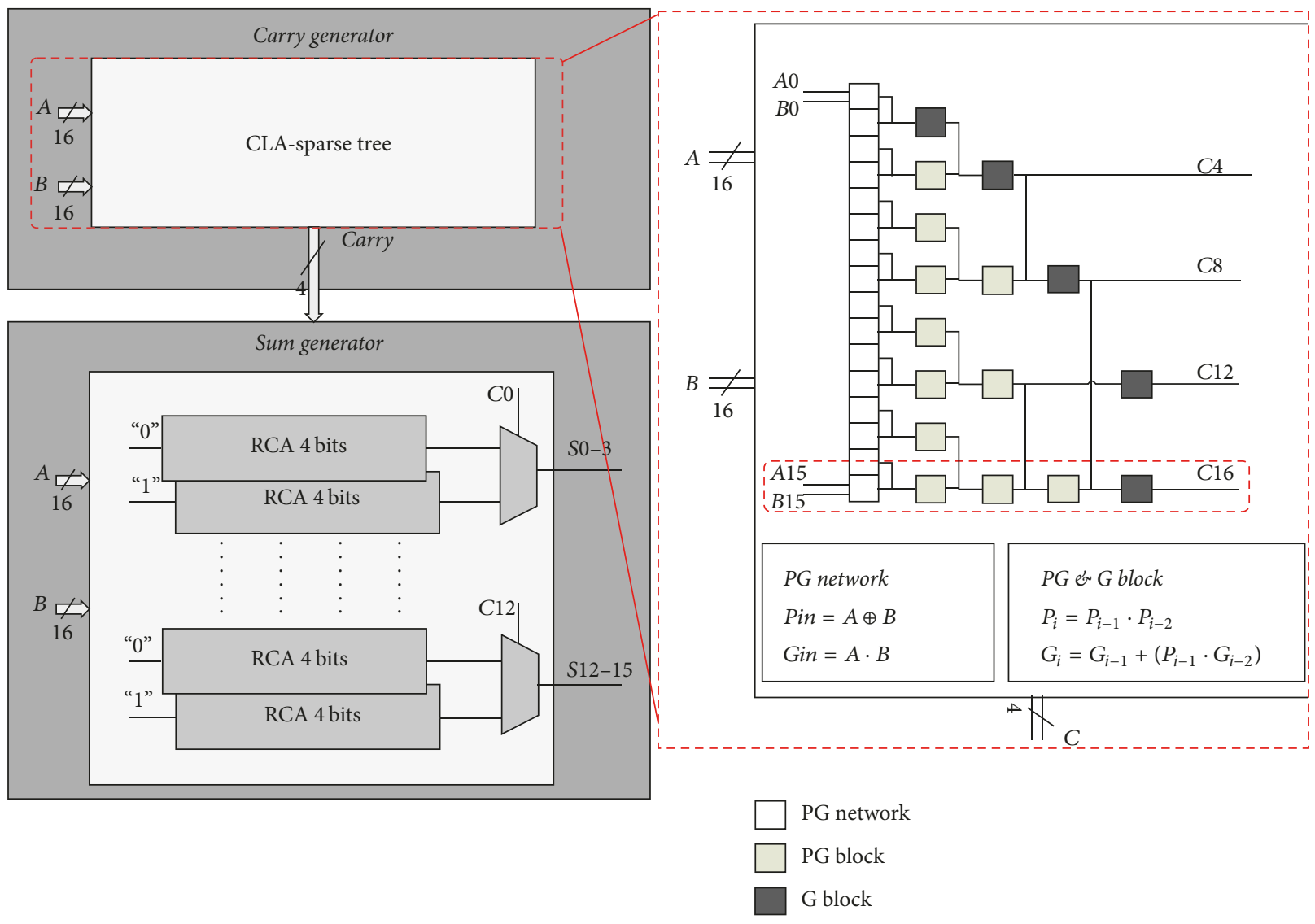

FIGURE 16: The general block diagram of the P4 adder and the carry generator block of P4 adder consists of a sparse tree.

\begin{tabular}{|c|c|c|c|c|}
\hline$\sqrt{1111}$ & X1011 & $X 1111$ & $\times 1011$ & Input- $A$ \\
\hline 0001 & & $X 0101$ & & Input- $B$ \\
\hline 0001 & X1101 & $X 0101$ & $X 0001$ & Sout: SE-LUT \\
\hline 0001 & 1101 & $X 0101$ & $\times 0001$ & Sout: FBE \\
\hline 0001 & X1101 & $\times 0101$ & $\times 0001$ & 田 Sout: TSB \\
\hline
\end{tabular}

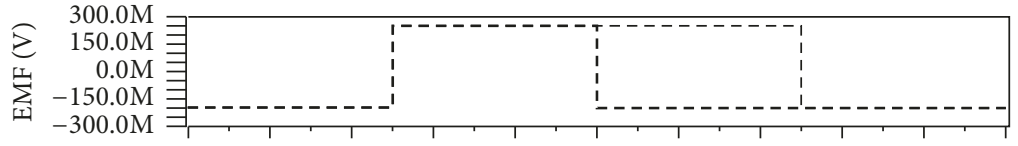

- - V(:tb_SE-LUT:s[2])

- - - V(:tb_SE-LUT:s[3])

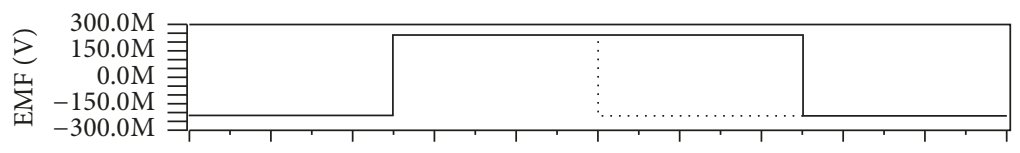

— $V($ :tb_FBE:s[2])

$V\left(: t b \_F B E: s[3]\right)$

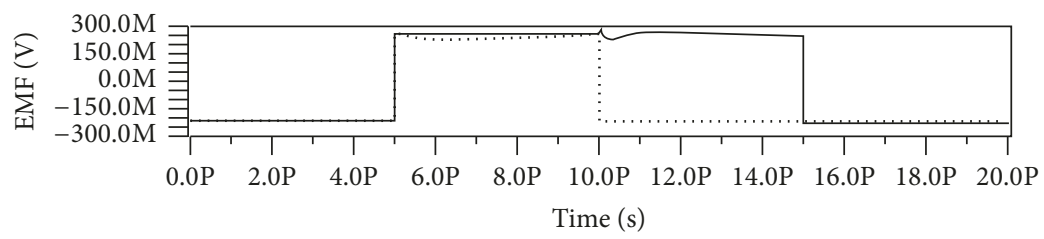

- V(:tb_TSB:s[2])

$V\left(: t b \_T S B: s[3]\right)$

FIGURE 17: Carry select adder output waveforms. 


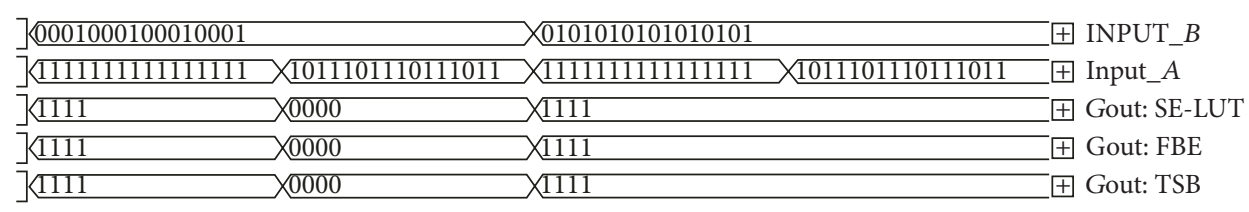
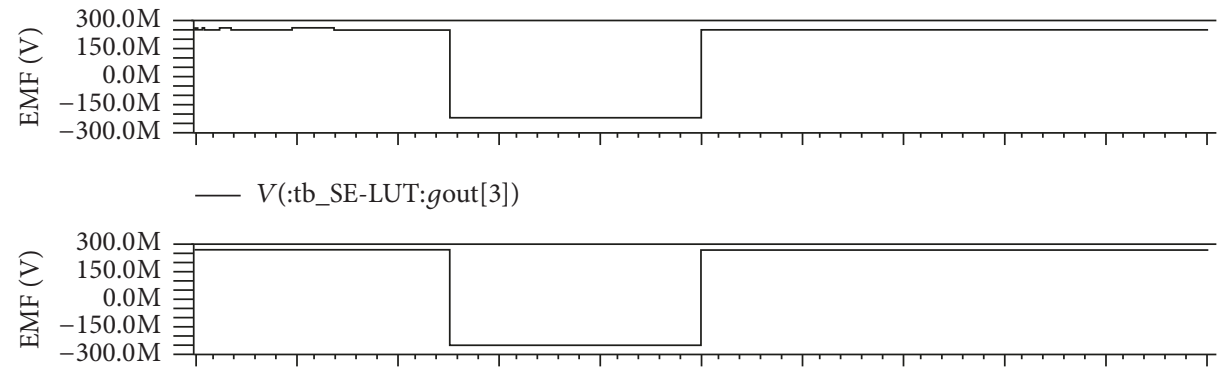

— V(:tb_FBE:gout[3])

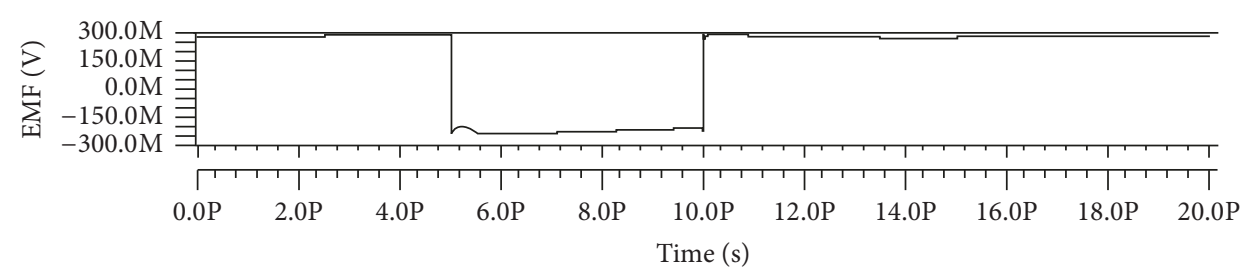

— V(:tb_TSB:gout[3])

FIGURE 18: Output waveforms for the sparse tree adder.

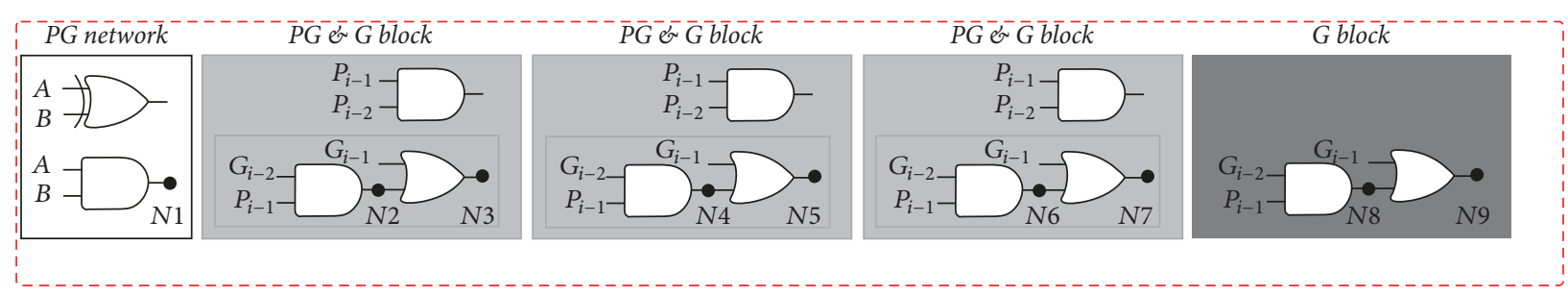

(a)

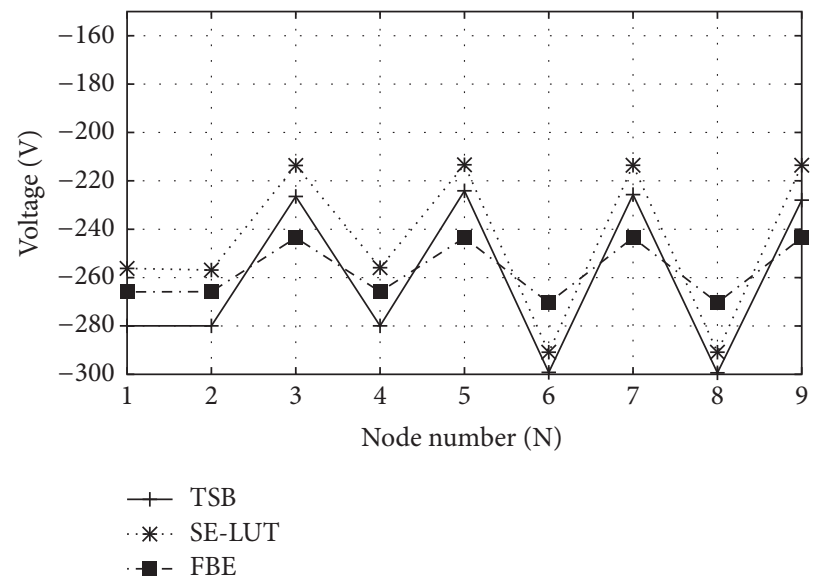

(b)

FIgURE 19: (a) A schematic of sparse tree area highlighted in Figure 16. Voltage level at each selected node (N1 to N9) of the sparse tree. 


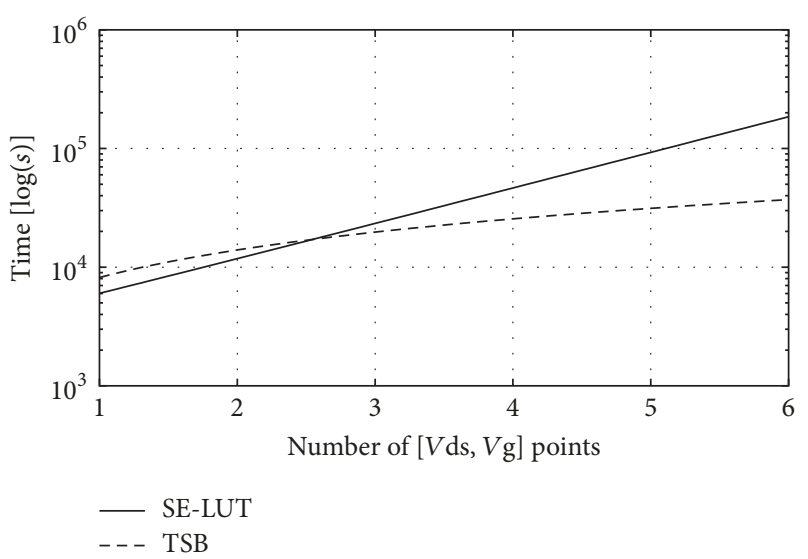

FIgURE 20: Timing comparison of SE-LUT and TSB model.

the number of iterations is large then the time would be large. In our case, we take the average time to calculate current (i.e., total time/total number of $V$ ds and $V g$ points). In this case, $T_{\text {Atm }}=2891 \mathrm{~s}$.

$T_{\text {Arch }}$ is the time required to implement the architecture in VHDL-ams. In all our simulations, implementation of P4 architecture by semiempirical lookup table requires less time as compared to other models. For transmission spectrum based model the time is about ten times the SE-LUT model, and $T_{\text {Arch }}($ for SE - LUT $)=240 \mathrm{~s}$.

$T_{\text {Intr }}$ is the time required for interpolation of SE-LUT model: this time is estimated as about 1 millisecond. $T_{\text {Algo }}$ is the time required for implementation of algorithm by TSB model in our case $T_{\text {Algo }}>1.35 e-3 \mathrm{~s}$.

Using these values in the equations we obtain the trend in Figure 20. The time required to implement the whole set of data and simulation for SE-LUT model increases exponentially, while the time of the TSB increases linearly. Thus, when $N V$ ds $<2$ SE-LUT requires a smaller amount of total time. However, after NVds $>2$ the total time of SELUT increases as compared to TBS. Thus in more realistic set of values, the computational demand of SE-LUT model will be much higher as compared to TSB model.

\section{Remarks and Conclusions}

In this work, we presented a modular framework based on VHDL-AMS for the description of circuits based on molecular-FET. The $I-V$ characteristics obtained by the three models proposed were compared to atomistic simulations. The three models used in the framework are transmission spectrum based model (TSB), FET based equivalent model (FBE), and semiempirical look-up table based model (SELUT). Though $I-V$ characteristics showed good results, the three models differ for flexibility and effort required to generate the necessary data. The framework allows the description of complex molecular circuits that have never been previously designed and tested in terms of functionality. In this case we adopted a crossbar based circuit and implemented a 16bit tree adder reproducing the architecture of the Pentium 4 adder. This is not at all possible using atomistic level simulations. We demonstrated the correct functionality and estimated the timing requirements to achieve the results if we consider to have a new molecule or new conditions on a previously available molecule. Results show that the SELUT model is straightforward for the setup and simple for the simulation but might require very long time in case a realistic set of data is necessary. The other models are more complex to be described and require some initial effort but are more flexible. In particular, TSB model easily allows including parameters variation derived by technological or environmental conditions for the molecule.

We have simulated, for the first time, the molecular transistor on a circuit level using the OPV molecule. Our approach allows fast assessment of the performance overcoming the need of complex quantum simulations of the system with many molecular devices currently not possible. Our methods allow seeing the impact of physics and chemistry of molecular devices on the applications and giving feedback to the technologists. In the future we aim to include the effect of process variations into the models. Some examples of the process variations include different binding strength between anchoring group and electrodes, stacking of molecules, and variation in molecular structure during synthesis.

\section{Conflicts of Interest}

The authors declare no conflicts of interest.

\section{Acknowledgments}

The authors wish to thank professor Paolo Lugli for his support and for allowing them to use the FBE model.

\section{References}

[1] S. Datta, Quantum Transport: Atom to Transistor, Cambridge University Press, 2005.

[2] G. Csaba and P. Lugli, "Read-out design rules for molecular crossbar architectures," IEEE Transactions on Nanotechnology, vol. 8, no. 3, pp. 369-374, 2009.

[3] A. Cao, E. J. R. Sudhölter, and L. C. P. M. de Smet, "Silicon nanowire-based devices for gas-phase sensing," Sensors, vol. 14, no. 1, pp. 245-271, 2013.

[4] A. Chiolerio, P. Allia, and M. Graziano, "Magnetic dipolar coupling and collective effects for binary information codification in cost-effective logic devices," Journal of Magnetism and Magnetic Materials, vol. 324, no. 19, pp. 3006-3012, 2012.

[5] M. T. Björk, H. Schmid, J. Knoch, H. Riel, and W. Riess, "Donor deactivation in silicon nanostructures," Nature Nanotechnology, vol. 4, no. 2, pp. 103-107, 2009.

[6] M. Vacca, J. Wang, M. Graziano, M. R. Roch, and M. Zamboni, "Feedbacks in QCA: A Quantitative Approach," IEEE Transactions on Very Large Scale Integration (VLSI) Systems, vol. 23, no. 10, pp. 2233-2243, 2015.

[7] S. Hong, R. Reifenberger, W. Tian, S. Datta, J. I. Henderson, and C. P. Kubiak, "Molecular conductance spectroscopy of conjugated, phenyl-based molecules on $\mathrm{Au}(111)$ : the effect of end groups on molecular conduction," Superlattices and Microstructures, vol. 28, no. 4, pp. 289-303, 2000.

[8] T. Ishida, W. Mizutani, N. Choi, U. Akiba, M. Fujihira, and H. Tokumoto, "Structural Effects on Electrical Conduction of Conjugated Molecules Studied by Scanning Tunneling Microscopy," 
The Journal of Physical Chemistry B, vol. 104, no. 49, pp. 11680$11688,2000$.

[9] H. C. Seong, B. Kim, and C. D. Frisbie, "Electrical resistance of long conjugated molecular wires," Science, vol. 320, no. 5882, pp. 1482-1486, 2008.

[10] F. Zahid, M. Paulsson, E. Polizzi, A. W. Ghosh, L. Siddiqui, and S. Datta, "A self-consistent transport model for molecular conduction based on extended Hückel theory with full threedimensional electrostatics," The Journal of Chemical Physics, vol. 123, no. 6, Article ID 064707, 2005.

[11] A. Mahmoud and P. Lugli, "Designing the rectification behavior of molecular diodes," Journal of Applied Physics, vol. 112, no. 11, Article ID 113720, 2012.

[12] M. Graziano, A. Pulimeno, R. Wang, X. Wei, M. R. Roch, and G. Piccinini, "Process variability and electrostatic analysis of molecular QCA," ACM Journal on Emerging Technologies in Computing Systems, vol. 12, no. 2, article no. 18, 2015.

[13] A. Pulimeno, M. Graziano, A. Sanginario, V. Cauda, D. Demarchi, and G. Piccinini, "Bis-ferrocene molecular QCA Wire: Ab initio simulations of fabrication driven fault tolerance," IEEE Transactions on Nanotechnology, vol. 12, no. 4, pp. 498-507, 2013.

[14] M. A. Reed, H. Song, and T. Lee, "Molecular Transistors," Emerging Nanoelectronic Devices, pp. 194-226, 2015.

[15] W. D. Wheeler and Y. Dahnovsky, "Molecular transistors with perpendicular gate field architecture: A strong gate field effect," The Journal of Physical Chemistry C, vol. 113, no. 3, pp. 10881092, 2009.

[16] T. M. Perrine, R. G. Smith, C. Marsh, and B. D. Dunietz, "Gating of single molecule transistors: Combining field-effect and chemical control," The Journal of Chemical Physics, vol. 128, no. 15, Article ID 154706, 2008.

[17] A. Zahir, A. Pulimeno, D. Demarchi et al., "Modular framework for molecular-FET device-to-circuit modeling," in Proceedings of the 15th IEEE International Conference on Nanotechnology, IEEE-NANO 2015, pp. 156-159, Italy, July 2015.

[18] Atomistix ToolKit, "Version 12.8.2," QuantumWise A/S, Copenhagen, Denmark, 2012.

[19] J. M. Soler, E. Artacho, J. D. Gale et al., "The SIESTA method for ab initio order-N materials simulation," Journal of Physics: Condensed Matter, vol. 14, no. 11, pp. 2745-2779, 2002.

[20] A. Zahir, A. Pulimeno, D. Demarchi et al., "EE-BESD: molecular FET modeling for efficient and effective nanocomputing design," Journal of Computational Electronics, vol. 15, no. 2, pp. 479-491, 2016.

[21] A. Zahir, S. A. A. Zaidi, A. Pulimeno et al., "Molecular transistor circuits: From device model to circuit simulation," in Proceedings of the 2014 IEEE/ACM International Symposium on Nanoscale Architectures, NANOARCH 2014, pp. 129-134, fra, July 2014.

[22] A. Mahmoud and P. Lugli, "Toward circuit modeling of molecular devices," IEEE Transactions on Nanotechnology, vol. 13, no. 3, pp. 510-516, 2014.

[23] A. Mahmoud and P. Lugli, "Atomistic study on dithiolated oligophenylenevinylene gated device," Journal of Applied Physics, vol. 116, no. 20, Article ID 204504, 2014.

[24] M. I. Schukfeh, K. Storm, A. Mahmoud et al., "Conductance enhancement of InAs/InP heterostructure nanowires by surface functionalization with oligo(phenylene vinylene)s," ACS Nano, vol. 7, no. 5, pp. 4111-4118, 2013.
[25] VHDL Analog and Mixed-Signal Extensions, IEEE Std 1076.11999.

[26] ADVance MS (ADMS), Reference Manual, Mentor Graphics, 2013.

[27] E. Christen and K. Bakalar, "VHDL-AMS - a hardware description language for analog and mixed-signal applications," IEEE Transactions on Circuits and Systems II: Analog and Digital Signal Processing, vol. 46, no. 10, pp. 1263-1272, 1999.

[28] P. Loumeau and J. F. Naviner, "VHDL-AMS behavioral modelling and simulation of high-pass delta-sigma modulator," in Proceedings of the BMAS 2005 - 2005 IEEE International Behavioral Modeling and Simulation Workshop, pp. 106-111, USA, September 2005.

[29] M. R. Casu, M. Crepaldi, and M. Graziano, "A VHDL-AMS simulation environment for an UWB impulse radio transceiver," IEEE Transactions on Circuits and Systems I: Regular Papers, vol. 55, no. 5, pp. 1368-1381, 2008.

[30] F. Pêcheux, C. Lallement, and A. Vachoux, "VHDL-AMS and Verilog-AMS as alternative hardware description languages for efficient modeling of multidiscipline systems," IEEE Transactions on Computer-Aided Design of Integrated Circuits and Systems, vol. 24, no. 2, pp. 204-224, 2005.

[31] M. Graziano and M. Ruo Roch, "An automotive CD-player electro-mechanics fault simulation using VHDL-AMS," Journal of Electronic Testing, vol. 24, no. 6, pp. 539-553, 2008. 


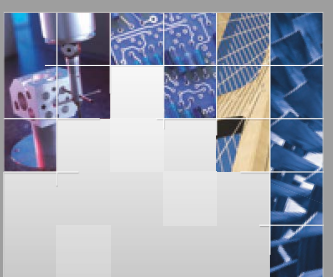

\section{Enfincering}
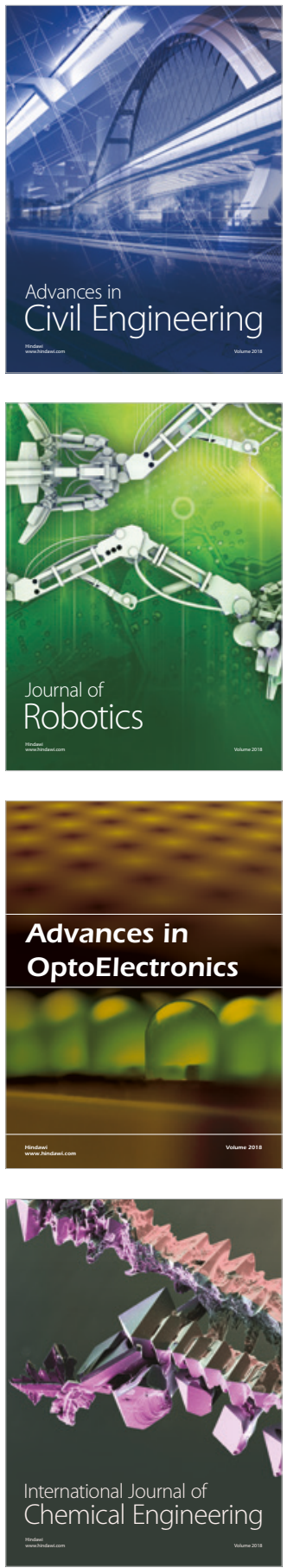

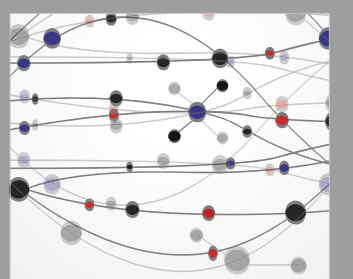

\section{Rotating \\ Machinery}

The Scientific World Journal

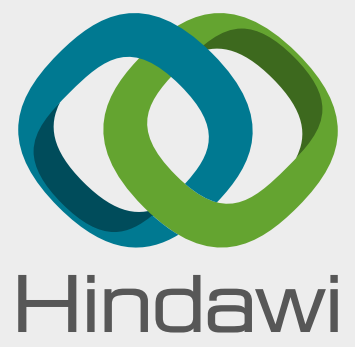

Submit your manuscripts at

www.hindawi.com
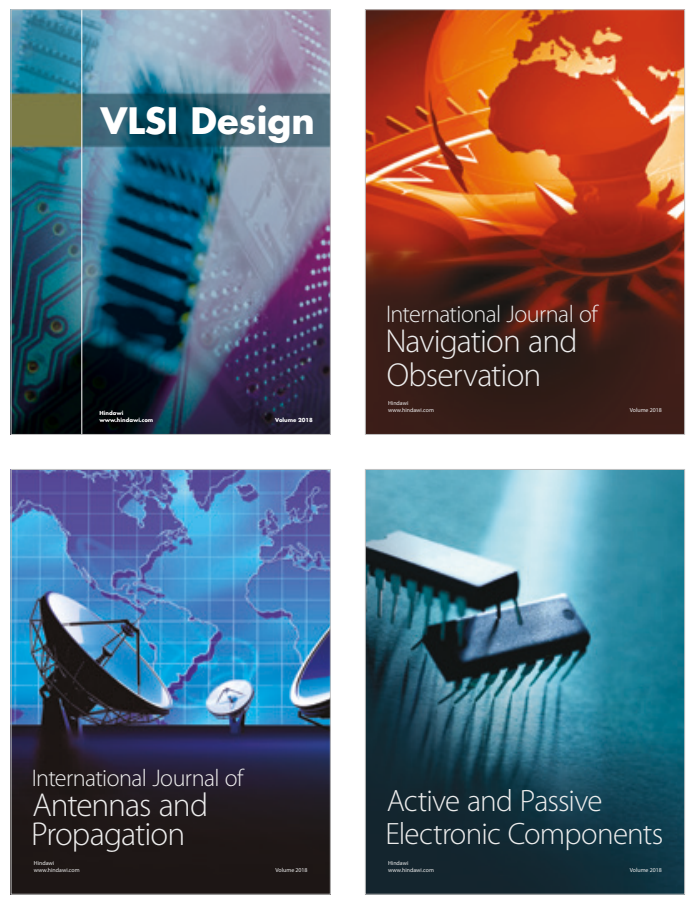
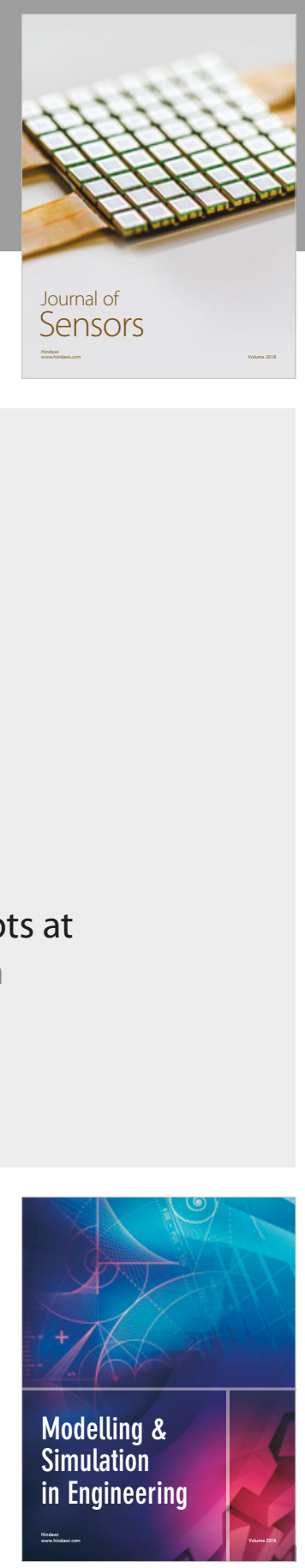

\section{Advances \\ Multimedia}
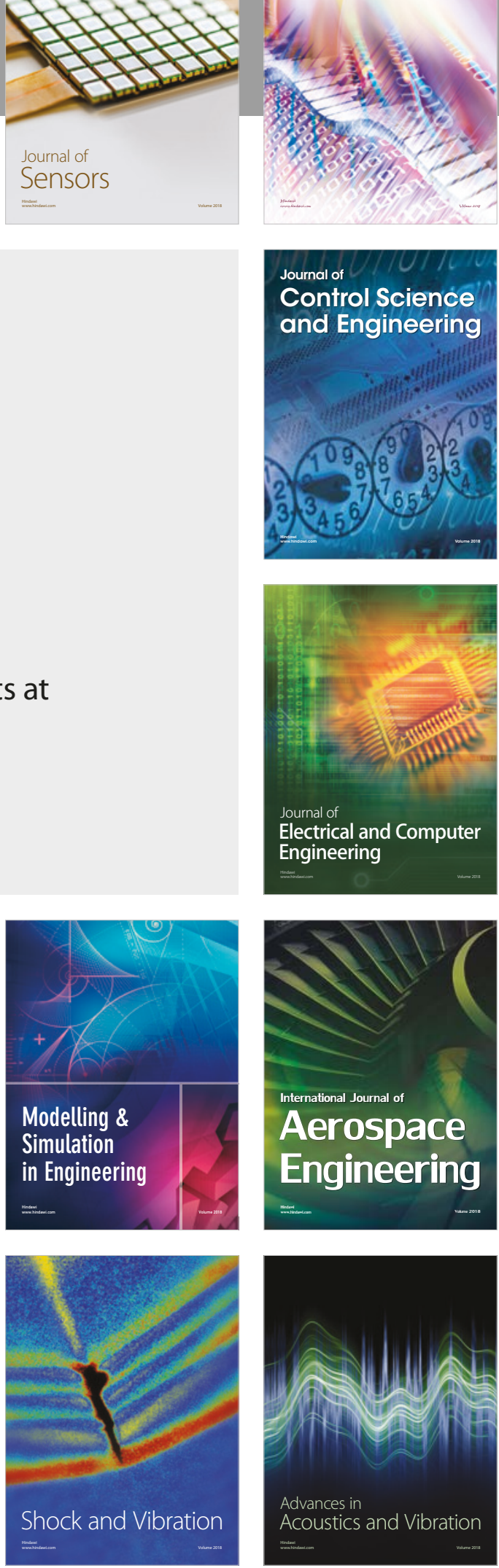